\title{
Inclusion of the Third Zonal Harmonic in an Accurate Reference Orbit of an Artificial Satellite*
}

\author{
John P. Vinti ${ }^{1}$
}

(November 17, 1965)

\begin{abstract}
The previous paper gave the general theory and physical principles involved in so modifying the author's spheroidal potential for an oblate planet as to permit exact inclusion of the effects of the third zonal harmonic of the planet's gravitational field. The present paper carries out the computational details necessary to derive the resulting orbit, which now corresponds to a potential fitted exactly through the third zonal harmonic and to about two-thirds of the fourth.

The accuracy of the orbit itself, as a solution for the given potential, depends on the accuracy of solution of a certain cubic equation. The paper works out this solution through terms of the third order in $J_{2}$, the coefficient of the second zonal harmonic, but its accuracy, and thus that of the secular terms, may be increased at will. Periodic terms are carried through the second order, but their accuracy may also be increased.

An obvious advantage of accounting for $J_{3}$ in this way is the absence of small denominators in $e$ or $\sin I$ that occur in a perturbation theory. Another is the resulting increase in accuracy, through terms in $J_{2}^{2}$, of the long-periodic third harmonic terms.
\end{abstract}

Key Words: Third zonal harmonic, increased accuracy, spheroidal reference orbit, artificial satellite.

\section{Introduction}

The previous paper ${ }^{2}$ [V1966] developed the theory and physical principles for inclusion of the third zonal harmonic of a planet's gravitational potential in an accurate reference orbit of an artificial satellite. The aim of the present paper is to work out the resulting generalized reference orbit in such detail as to permit practical calculation.

To begin, let $\rho, \eta, \varphi$ be oblate spheroidal coordinates, satisfying the equations

$$
\begin{aligned}
x+i y & =r \cos \theta \exp i \varphi=\left[\left(\rho^{2}+c^{2}\right)\left(1-\eta^{2}\right)\right]^{1 / 2} \exp i \varphi \\
z & =r \sin \theta=-\delta+\rho \eta .
\end{aligned}
$$

Here $x, y, z$ are rectangular coordinates of a satellite in a Cartesian frame, with origin $O$ at the center of mass of an oblate planet, $O z$ pointing along the polar axis, and $O x$ pointing toward its vernal equinox. Also $r, \theta, \varphi$ are the planetocentric distance, latitude, and right ascension.

If $r_{e}$ is the equatorial radius and if

$$
\begin{aligned}
& c_{2}=r_{e}^{2} J_{2}\left(1-\frac{1}{4} J_{3}^{2} J_{2}^{-3}\right) \\
& \delta=-\frac{1}{2} r_{e} J_{2}^{-1} J_{3},
\end{aligned}
$$

then the gravitational potential

$$
V=-\mu\left(\rho^{2}+c^{2} \eta^{2}\right)^{-1}(\rho+\eta \delta)
$$

leads to separability of the problem of satellite motion [V1966]. Here $\delta \approx 7 \mathrm{~km}$ for the earth and

\footnotetext{
${ }^{*}$ Research supported by the National Aeronautical and Space Administration, Washington, D.C.

'Present Adress: Department of Mathematics, North Carolina State University, Raleigh, North Carolina.

"Since there will be so many references to my own papers, I abbreviate my name to the initial $\mathrm{V}$ in the body of the paper
} 
the potential (4) leads to a fit of

$$
V=-\frac{\mu}{r}\left[1-\sum_{2}^{\infty}\left(\frac{r_{e}}{r}\right)^{n} J_{n} P_{n}(\sin \theta)\right]
$$

exactly through the third zonal harmonic. It also gives about two-thirds of the fourth harmonic and negligible values, of order $J_{2}^{3}$ or smaller, for the higher harmonics.

By V1959, if $\alpha_{1}$ is the energy, $\alpha_{3}$ the $z$-component of angular momentum, and $\alpha_{2}$ the separation constant, the generalized momenta $p_{\rho}, p_{\eta}$, and $p_{\varphi}$ are given by

$$
p_{\varphi}=\alpha_{3} \quad(6.1), \quad p_{\rho}= \pm\left(\rho^{2}+c^{2}\right)^{-1} F(\rho)^{1 / 2} \quad(6.2), \quad p_{\eta}= \pm\left(1-\eta^{2}\right)^{-1} G(\eta)^{1 / 2} .
$$

Here $F(\rho)$ and $G(\eta)$ are the quartics

$$
\begin{gathered}
F(\rho)=c^{2} \alpha_{3}^{2}+\left(\rho^{2}+c^{2}\right)\left(-\alpha_{2}^{2}+2 \mu \rho+2 \alpha_{1} \rho^{2}\right) \\
G(\eta)=-\alpha_{3}^{2}+\left(1-\eta^{2}\right)\left(\alpha_{2}^{2}+2 \mu \eta \delta+2 \alpha_{1} c^{2} \eta^{2}\right) .
\end{gathered}
$$

The Hamilton-Jacobi function $W(\rho, \eta, \varphi)$ is then

$$
W=\int p \varphi d \varphi+\int p_{\rho} d \rho+\int p \eta d \eta=\alpha_{3} \varphi+\int_{\rho_{1}}^{\rho} \pm\left(\rho^{2}+c^{2}\right)^{-1} F(\rho)^{1 / 2} d \rho+\int_{\eta_{1}}^{\eta} \pm\left(1-\eta^{2}\right)^{-1} G(\eta)^{1 / 2} d \eta .
$$

The quartic $F(\rho)$ has two physical zeros, $\rho_{1}$ and $\rho_{2}$, and the quartic $G(\eta)$ has two physical zeros, $\eta_{1}$ and $\eta_{0}$. The possible motions are thus confined to the toroidal region

$$
\begin{gathered}
0<\rho_{1} \leqq \rho \leqq \rho_{2} \\
-1 \leqq \eta_{1} \leqq \eta \leqq \eta_{0} \leqq 1 .
\end{gathered}
$$

If $\beta_{1}, \beta_{2}^{\prime}$, and $\beta_{3}^{\prime}$ are constants, the orbit is then given by

$$
t+\beta_{1}=\frac{\partial W}{\partial \alpha_{1}}, \quad \beta_{2}^{\prime}=\frac{\partial W}{\partial \alpha_{2}}, \quad \beta_{3}^{\prime}=\frac{\partial W}{\partial \alpha_{3}}
$$

With $\rho_{1}$ and $\eta_{1}$ as lower limits of the integrals in (8), these become

$$
\begin{aligned}
t+\beta_{1} & =R_{1}+c^{2} N_{1} \\
\beta_{2}^{\prime} & =-\alpha_{2} R_{2}+\alpha_{2} N_{2} \\
\varphi & =\beta_{3}^{\prime}-c^{2} \alpha_{3} R_{3}+\alpha_{3} N_{3} .
\end{aligned}
$$

Here the $\rho$-integrals are given by

$$
R_{1}=\int_{\rho_{1}}^{\rho} \pm \rho^{2} F^{-1 / 2} d \rho, \quad R_{2}=\int_{\rho_{1}}^{\rho} \pm F^{-1 / 2} d \rho, \quad R_{3}=\int_{\rho_{1}}^{\rho} \pm\left(\rho^{2}+c^{2}\right)^{-1} F^{-1 / 2} d \rho
$$

and the $\eta$-integrals by

$$
\begin{aligned}
& N_{1}=\int_{\eta_{1}}^{\eta} \pm \eta^{2} G^{-1 / 2} d \eta \\
& N_{2}=\int_{\eta_{1}}^{\eta} \pm G^{-1 / 2} d \eta
\end{aligned}
$$




$$
N_{3}=\int_{\eta_{1}}^{\eta} \pm\left(1-\eta^{2}\right)^{-1} G^{-1 / 2} d \eta
$$

With $\eta_{1}$ as lower limit in the $\eta$-integrals, rather than 0 as before, we shall find that in the Keplerian limit $\left(J_{2} \rightarrow 0, J_{3} \rightarrow 0\right)$ the constant $-\beta_{1}$ will again reduce to the time of passage through perigee. The constant $\beta_{2}^{\prime}$, however, will not reduce to $\omega$, the argument of perigee, but to $\omega+\pi / 2$. Similarly $\beta_{3}^{\prime}$ will not reduce to $\Omega$, the right ascension of the ascending node, but to $\Omega-\frac{1}{2} \pi \operatorname{sgn} \alpha_{3}$.

The $\rho$-integrals have already been worked out [V1961b]. The new $\eta$-integrals, however, will contain $\delta$, involving $J_{3}$, and will thus have to be reevaluated. The task of the present paper is thus to reevaluate these $\eta$-integrals $N_{1}, N_{2}$, and $N_{3}$, insert them into (11), invert (11.1) and (11.2) to find $\rho$ and $\eta$ as functions of time, and then insert the results into (11.3) to find $\varphi$ as a function of time. We shall then have the new reference orbit, corresponding to the generalized potential (4) that allows for exact inclusion of the third harmonic.

\section{Factoring the Quartics}

In order to evaluate the integrals, we first have to factor the quartics $F(\rho)$ and $G(\eta)$. The factoring of $F(\rho)$ was essentially done in V1961b and the only new task that we have in connection with it is to relate associated quantities to the new orbital elements, which will depend on the new factoring of $G(\eta)$. To factor $G(\eta)$ note that by (7.2)

$$
G(\eta)=\alpha_{2}^{2}-\alpha_{3}^{2}+2 \mu \eta \delta+\left(2 \alpha_{1} c^{2}-\alpha_{2}^{2}\right) \eta^{2}-2 \mu \eta^{3} \delta-2 \alpha_{1} c^{2} \eta^{4}
$$

By (6.3) and (13), if the orbit ever reaches $\eta=0$, we should have $\alpha_{2}^{2}-\alpha_{3}^{2} \geqq 0$. If indeed the odd harmonics are all put equal to zero, we should have $\delta=\frac{1}{2} r_{e} J_{2}^{-1}\left|J_{3}\right|=0$ and by (1.2) a motion $z=0$ in the equatorial plane would be possible, with $\eta$ remaining zero and $\alpha_{2}^{2}-\alpha_{3}^{2} \geqq 0$.

As soon as we insert the third harmonic, however, there arises a doubt that $\eta=0$ will always be reached. Accordingly, we must leave the sign of $\alpha_{2}^{2}-\alpha_{3}^{2}$ open. For bounded orbits we now have $\alpha_{1}<0$ and the table

\begin{tabular}{r|l}
\hline \hline$\eta$ & \multicolumn{1}{|c}{$G(\eta)$} \\
\hline$-\infty$ & $+\infty$ \\
-1 & $-\alpha_{3}^{2} \leqq 0$ \\
0 & $\alpha_{2}^{2}-\alpha_{3}^{2}$, sign either \pm \\
+1 & $-\alpha_{3}^{2} \leqq 0$ \\
$+\infty$ & $+\infty$ \\
\hline
\end{tabular}

There are thus two non-physical zeros, between $-\infty$ and -1 and between +1 and $+\infty$, and two physical zeros, between -1 and +1 . Call these latter $\eta_{1}$ and $\eta_{0}$, with $\eta_{1} \leqq \eta_{0}$. If $\delta=0, G(\eta)$ becomes a quadratic in $\eta^{2}$ and $\eta_{1}=-\eta_{0}$, where $\eta_{0} \geqq 0$, the equality sign holding when $\alpha_{2}^{2}-\alpha_{3}^{2}=0$.

To see what happens when $\delta \neq 0$, make a plot of $G(\eta)$ versus $\eta$, noting that $G^{\prime}(0)=2 \mu \delta>0$. In order to avoid more than two zeros between $\eta=-1$ and $\eta=+1$, we must then have 


$$
\begin{array}{ccc}
\text { if } & \text { that } & \text { and } \\
\alpha_{2}^{2}-\alpha_{3}^{2}>0 & \eta_{1}<0<\eta_{0} & -\eta_{0} \eta_{1}>0 \\
\alpha_{2}^{2}-\alpha_{3}^{2}=0 & 0=\eta_{1}<\eta_{0} & -\eta_{0} \eta_{1}=0 \\
\alpha_{2}^{2}-\alpha_{3}^{2}<0 & 0<\eta_{1} \leqq \eta_{0} & -\eta_{0} \eta_{1}<0
\end{array}
$$

The paper V1961b indicated the following form for factoring $G(\eta)$ :

$$
G(\eta)=\left(\alpha_{2}^{2}-\alpha_{3}^{2}\right)\left(1-\frac{\eta}{\eta_{0}}\right)\left(1-\frac{\eta}{\eta_{1}}\right)\left(1+C_{1} \eta-C_{2} \eta^{2}\right) .
$$

Here, since $\eta_{1} \leqq \eta \leqq \eta_{0}$, we have

$$
\left(\eta-\eta_{1}\right)\left(\eta_{0}-\eta\right) \geqq 0 .
$$

For $\alpha_{2}^{2}-\alpha_{3}^{2}>0$, we have $-\eta_{0} \eta_{1}>0$ and division gives

$$
\left(1-\frac{\eta}{\eta_{0}}\right)\left(1-\frac{\eta}{\eta_{1}}\right) \geqq 0
$$

For $\alpha_{2}^{2}-\alpha_{3}^{2}<0$, we have $\eta_{0} \eta_{1}>0$ and division gives

$$
\left(1-\frac{\eta}{\eta_{0}}\right)\left(1-\frac{\eta}{\eta_{1}}\right)<0
$$

In either case, since $G(\eta) \geqq 0$, we find that the factor $1+C_{1} \eta-C_{2} \eta^{2}>0$, where $C_{1}$ and $C_{2}$ will both be small compared to unity.

On comparing coefficients in (13) and (14), with

$$
\begin{aligned}
& q_{0} \equiv \mu^{-1}\left(\alpha_{2}^{2}-\alpha_{3}^{2}\right) \\
& P \equiv \frac{1}{2}\left(\eta_{0}+\eta_{1}\right) \\
& S \equiv-\eta_{0} \eta_{1},
\end{aligned}
$$

and with the relations from V1961b, viz,

$$
\begin{gathered}
a_{0} \equiv-\frac{1}{2} \frac{\mu}{\alpha_{1}}, \quad e_{0} \equiv\left(1+\frac{2 \alpha_{1} \alpha_{2}^{2}}{\mu^{2}}\right)^{1 / 2}, \quad p_{0} \equiv a_{0}\left(1-e_{0}^{2}\right) \\
\alpha_{2}^{2}=\mu p_{0},
\end{gathered}
$$

we have

$$
\begin{gathered}
2 P+C_{1} S=\frac{2 \delta}{q_{0}} S \\
1+C_{2} S-2 C_{1} P=\frac{p_{0}+c^{2} / a_{0}}{q_{0}} S \\
C_{1}+2 C_{2} P=\frac{2 \delta}{q_{0}} S
\end{gathered}
$$




$$
C_{2}=\frac{c^{2}}{a_{0} q_{0}} S
$$

Along with three constants, to be derived later from $\beta_{1}, \beta_{2}^{\prime}$, and $\beta_{3}^{\prime}$, we shall choose as orbital elements

$$
\begin{aligned}
& a=\frac{1}{2}\left(\rho_{1}+\rho_{2}\right) \\
& e=\frac{\rho_{2}-\rho_{1}}{\rho_{2}+\rho !} \\
& p \equiv a\left(1-e^{2}\right)
\end{aligned}
$$

as in V1961b, and

$$
S \equiv-\eta_{0} \eta_{1}
$$

If $\delta=0, \eta_{1}$ becomes $-\eta_{0}$, and $S$ reduces to the old $\eta_{0}^{2}=\sin ^{2} I$ of V1961b. Note that now, however, the new orbital element $S$ can sometimes become negative, viz, for orbits sufficiently close to equatorial. Note that for a polar orbit $\eta_{0}=+1$ and $\eta_{1}=-1$, so that $S$ then equals unity. In any case, since $-1 \leqq \eta_{1} \leqq \eta_{0} \leqq 1$, we have

$$
S^{2} \leqq 1
$$

Our next task is to express the factorization of the quartics $F(\rho)$ and $G(\eta)$ completely in terms of the elements $a, e$, and the new $S$. To do so, we must first express $C_{1}, C_{2}$, and $P$ in terms of $c, \delta, a_{0}, p_{0}$, and $S$; that is, we must eliminate $q_{0}$.

On inserting (22.4) into (22.3) and solving the resulting equation simultaneously with (22.1), we find

$$
\begin{aligned}
C_{1} & =\left(1-\frac{c^{2}}{a_{0} q_{0}} S^{2}\right)^{-1} \frac{2 \delta}{q_{0}} S\left(1-\frac{c^{2}}{a_{0} q_{0}} S\right) \\
P & =\left(1-\frac{c^{2}}{a_{0} q_{0}} S^{2}\right)^{-1} \frac{\delta}{q_{0}} S(1-S) .
\end{aligned}
$$

If we now insert (25.1), (25.2), and (22.4) into (22.2) and call

$$
\frac{p_{0}}{q_{0}} S \equiv u
$$

we find

$$
\frac{1}{u}=1+\frac{c^{2}}{a_{0} p_{0}}(1-S)+\frac{\left(\frac{2 \delta}{p_{0}}\right)^{2}(1-S)\left(\frac{1}{u}-\frac{c^{2}}{a_{0} p_{0}}\right)}{\left(\frac{1}{u}-\frac{c^{2}}{a_{0} p_{0}} S\right)^{2}} .
$$

For $\delta=0, S$ becomes $\sin ^{2} I, u$ becomes $\sin ^{2} I / \sin ^{2} i_{0}$, where

$$
i_{0} \equiv \cos ^{-1} \frac{\alpha_{3}}{\alpha_{2}}
$$


from $V 1961 b$, and (27) reduces to

$$
\sin ^{2} i_{0}=\sin ^{2} I+\frac{c^{2}}{a_{0} p_{0}} \sin ^{2} I \cos ^{2} I,
$$

in agreement with (4.8) of V1961b. Equation (27) is a cubic in $u^{-1}$, but we may solve it by successive approximations. To do so, note first that

$$
\frac{1}{u}=1+\frac{c^{2}}{a_{0} p_{0}}(1-S)+O\left(\delta^{2}\right)
$$

If we insert (30) into the right side of (27), the error will be of order $\delta^{4}$, i.e., of order $J_{2}^{4}$. Thus

$$
\frac{1}{u}=1+\frac{c^{2}}{a_{0} p_{0}}(1-S)+\epsilon,
$$

where

$$
\epsilon=\frac{\left(\frac{2 \delta}{p_{0}}\right)^{2}(1-S)\left(1-\frac{c^{2}}{a_{0} p_{0}} S\right)}{\left[1+\frac{c^{2}}{a_{0} p_{0}}(1-2 S)\right]^{2}}+O\left(J_{2}^{4}\right)
$$

(Insertion of (31) in the right side of (27) would reduce the error to $O\left(J_{2}^{6}\right)$, but such accuracy is not needed.) Equation (31) frees $u$ from $q_{0}$ and thus also $C_{1}, C_{2}$, and $P$, since by (22.4), (25), and (26)

$$
\begin{aligned}
C_{2} & =\frac{c^{2}}{a_{0} p_{0}} u \\
C_{1} & =\left(1-\frac{c^{2}}{a_{0} p_{0}} S u\right)^{-1} \frac{2 \delta}{p_{0}} u\left(1-\frac{c^{2}}{a_{0} p_{0}} u\right) \\
P & =\left(1-\frac{c^{2}}{a_{0} p_{0}} S u\right)^{-1} \frac{\delta}{p_{0}} u(1-S) .
\end{aligned}
$$

These quantities $C_{1}, C_{2}$, and $P$ are then correct through order $J_{2}^{4}$.

Since $|S| \leqq 1$, we find from (31) that $\epsilon \geqq 0$ and $u^{-1} \geqq 1$. Thus

$$
0<u \leqq 1
$$

and then, by (32)

$$
C_{2}>0, \quad C_{1}>0, \quad P \geqq 0 .
$$

In order to obtain all the required quantities as functions of $a, e$, and $S$, we must now summarize the factoring of $F(\rho)$, given in V1961b. We have

$$
\begin{gathered}
F(\rho)=-2 \alpha_{1}\left(\rho-\rho_{1}\right)\left(\rho_{2}-\rho\right)\left(\rho^{2}+A \rho+B\right) \\
a \equiv \frac{1}{2}\left(\rho_{1}+\rho_{2}\right), \quad e \equiv \frac{\rho_{2}-\rho_{1}}{\rho_{2}+\rho_{1}}, \quad p \equiv a\left(1-e^{2}\right) .
\end{gathered}
$$


Then, with $q_{0}$ defined by (18) and $a_{0}$ and $p_{0}$ by (21), comparison of coefficients in (34.1) and (7.1) gives

$$
\begin{aligned}
2 a-A & =2 a_{0} \\
B+a p-2 A a & =c^{2}+a_{0} p_{0} \\
2 a B-A a p & =2 a_{0} c^{2} \\
B a p & =a_{0} p_{0} c^{2} q_{0} / p_{0} .
\end{aligned}
$$

We now regard $a, p, S, c$, and $\delta$ as known and have to solve the eight equations in (35), (32), and (26) for the eight unknowns $a_{0}, p_{0}, q_{0}, A, B, C_{1}, C_{2}$, and $P$. Here $u$ is an auxiliary parameter given by (31).

From (35.4), (35.2), (26), and (31), we find

$$
B a p=c^{2} S\left[B+a p-2 A a-c^{2} S+\frac{4 \frac{a_{0}}{p_{0}} \delta^{2}(1-S)\left(1-\frac{c^{2}}{a_{0} p_{0}} S\right)}{\left[1+\frac{c^{2}}{a_{0} p_{0}}(1-2 S)\right]^{2}}+O\left(J_{2}^{4}\right)\right]
$$

Now since $A$ and $B$ are both of order $J_{2}$, we have from (35.1) and (35.2)

$$
\begin{gathered}
\frac{c^{2}}{a_{0} p_{0}}=\frac{c^{2}}{a p}+O\left(J_{2}^{2}\right) \\
\frac{a_{0}}{p_{0}}=\frac{a}{p}\left(1+\frac{c^{2}}{a p}+\frac{2 A}{p}-\frac{A}{a}-\frac{B}{a p}\right)+O\left(J_{2}^{2}\right)
\end{gathered}
$$

Because of the factor $\delta^{2}$, of order $J_{2}^{2}$, insertion of (37) into (36) will not change the order of the error. Thus

$$
B a p=c^{2} S\left[B+a p-2 A a-c^{2} S+\frac{4 a}{p}(1-S) \delta^{2}\left\{1+\frac{c^{2}}{a p}(3 S-1)+A\left(\frac{2}{p}-\frac{1}{a}\right)-\frac{B}{a p}\right\}\right]+O\left(J_{2}^{5}\right) .
$$

This is an equation involving only $A, B$, and "known" quantities. Another follows by combination of (35.1) and (35.3). It is

$$
2 a B-A a p=(2 a-A) c^{2} .
$$

The solution of (38) and (39) for $A$ and $B$ is

$$
\begin{gathered}
A=\frac{-2 a c^{2}\left(a p-c^{2} S\right)(1-S)+\frac{8 a^{2} c^{2}}{p} \delta^{2}\left\{1+\frac{c^{2}}{a p}(3 S-2)\right\} S(1-S)}{\left(a p-c^{2}\right)\left(a p-c^{2} S\right)+4 a^{2} c^{2} S+\frac{4 c^{2}}{p^{2}} \delta^{2}\left(3 a p-4 a^{2}-c^{2}\right) S(1-S)} \\
B=(2 a)^{-1}\left(a p-c^{2}\right) A+c^{2} .
\end{gathered}
$$


For a polar orbit, for which $S=1$, we have from (40) and (35)

$$
A=0 \quad B=c^{2} \quad a_{0}=a \quad p_{0}=p .
$$

For such a polar orbit, if $e=0$, we then have

$$
\rho=a=a_{0}=p=p_{0}, \quad e_{0}=0 .
$$

Equations (40) give us $A$ and $B$ in terms of known quantities. To find the others, note that $a_{0}$ then follows from (35.1), $p_{0}$ from (35.2), $q_{0}$ from (26) and (31), and $C_{2}, C_{1}$, and $P$ from (32). With use of these equations, the errors arising from the approximate solution of the cubic (27) are of order $J_{2}^{5}$ for all the unknowns except $q_{0}$. For $q_{0}$ the error is of order $J_{2}^{4}$. The "known" quantities are $c, \delta, a, p$, and $S$. Here $c=r_{e} J_{2}^{1 / 2}\left(1-\frac{1}{4} J_{3}^{2} J_{2}^{-3}\right)^{1 / 2}, \delta=\frac{1}{2} r_{e} J_{2}^{-1}\left|J_{3}\right|, a$ is a constant "semi-major axis," $p$ a constant "semi-latus rectum," and $S$ a constant quantity analogous to the squared sine of the inclination for inclinations not too close to zero. For inclinations close to zero, however, $S$ may be negative for $\delta \neq 0$.

\section{The $\eta$-Integrals}

From (14), (18), (19), and (20), we find

$$
G(\eta)=\mu q_{0} S^{-1}\left(S+2 P \eta-\eta^{2}\right)\left(1+C_{1} \eta-C_{2} \eta^{2}\right) .
$$

Now

$$
S+2 P \eta-\eta^{2} \equiv P^{2}+S-(\eta-P)^{2} .
$$

The physical zeros $\eta_{1}$ and $\eta_{0}$ of $G(\eta)$ are the zeros of (44), viz,

$$
\begin{aligned}
& \eta_{0}=P+\left(P^{2}+S\right)^{1 / 2} \\
& \eta_{1}=P-\left(P^{2}+S\right)^{1 / 2} .
\end{aligned}
$$

For $\delta \neq 0, S$ may be negative, with smallest possible value $S=-P^{2}$, corresponding to

$$
\eta_{0}=\eta_{1}=\eta^{\prime}=\frac{\delta}{p_{0}+c^{2} / a_{0}}\left(1-\frac{3 \delta^{2}}{p_{0}^{2}}\right)+O\left(J_{2}^{4}\right)
$$

from V1966.

With

$$
Q \equiv\left(P^{2}+S\right)^{1 / 2} \geqq 0,
$$

we may now introduce a variable $\psi$, such that

$$
\eta=P+Q \sin \psi
$$

If $\psi$ continually increases, so that $\dot{\psi}>0$ always, $\eta$ will then vary between the limits $\eta_{1}$ and $\eta_{0}$ and the double sign in eqs (12) for the $\eta$-integrals will disappear, with $\psi$ as integration variable. Thus $\psi$ is a uniformizing variable. 
Insertion of (48) into (43) then gives

$$
G(\eta)=\mu q_{0} Q^{2} S^{-1}\left(1+C_{1} \eta-C_{2} \eta^{2}\right) \cos ^{2} \psi .
$$

and the factor $\pm G^{-1 / 2} d \eta$ in the integrals (12) becomes

$$
\begin{aligned}
\pm G^{-1 / 2} d \eta & =\left(\frac{S}{\mu q_{0} Q}\right)^{1 / 2}\left(1+C_{1} \eta-C_{2} \eta^{2}\right)^{-1 / 2} d \psi \\
& =\alpha_{2}^{-1} u^{1 / 2}\left(1+C_{1} \eta-C_{2} \eta^{2}\right)^{-1 / 2} d \psi
\end{aligned}
$$

by (26) and (21.2).

\section{The Integral $N_{2}$}

By (12.2), (50), and (48) we then find

$$
\alpha_{2} u^{-1 / 2} N_{2}=\int_{-\frac{\pi}{2}}^{\psi}\left(1+C_{1} \eta-C_{2} \eta^{2}\right)^{-1 / 2} d \psi .
$$

Here $C_{1}$ and $C_{2}$ are both positive, of order $J_{2}$. To evaluate (51), we place

$$
h \equiv i C_{2}^{1 / 2} \eta=O\left(J_{2}\right), \quad \lambda \equiv \frac{1}{2} i C_{1} C_{2}^{-1 / 2}=O\left(J_{2}^{1 / 2}\right),
$$

so that

$$
1+C_{1} \eta-C_{2} \eta^{2} \equiv 1-2 \lambda h+h^{2} .
$$

Since $\lambda=O\left(J_{2}^{1 / 2}\right)$, it follows that $\left|\lambda \pm \sqrt{\lambda^{2}-1}\right|=O(1)$ and

$$
|h| \ll \text { smaller of }\left|\lambda \pm \sqrt{\lambda^{2}-1}\right| .
$$

Thus a Legendre expansion is legitimate, so that

$$
\left(1+C_{1} \eta-C_{2} \eta^{2}\right)^{-1 / 2}=\left(1-2 \lambda h+h^{2}\right)^{-1 / 2}=\sum_{n=0}^{\infty} h^{n} P_{n}(\lambda)=\sum_{n=0}^{\infty} i^{n} C_{2}^{n / 2} \eta^{n} P_{n}(\lambda) .
$$

Then (51) and (55) lead to

$$
\alpha_{2} u^{-1 / 2} N_{2}=\sum_{n=0}^{\infty} F_{n}\left(C_{1}, C_{2}\right) \int_{-\frac{\pi}{2}}^{\psi} \eta^{n} d \psi,
$$

where

$$
F_{n}\left(C_{1}, C_{2}\right) \equiv i^{n} C_{2}^{n / 2} P_{n}\left(\frac{1}{2} i C_{1} C_{2}^{-1 / 2}\right) \text {. }
$$

are real polynomials in $C_{1}$ and $C_{2}$. Through $F_{8}$ they are

$F_{0}=1, \quad F_{1}=-\frac{1}{2} C_{1}, \quad F_{2}=\frac{3}{8} C_{1}^{2}+\frac{1}{2} C_{2}, \quad F_{3}=-\frac{5}{16} C_{1}^{3}-\frac{3}{4} C_{1} C_{2}$, 
$F_{4}=\frac{35}{128} C_{1}^{4}+\frac{15}{16} C_{1}^{2} C_{2}+\frac{3}{8} C_{2}^{2}, \quad F_{5}=-\frac{63}{256} C_{1}^{5}-\frac{35}{32} C_{1}^{3} C_{2}-\frac{15}{16} C_{1} C_{2}^{2}$,

$F_{6}=\frac{231}{1024} C_{1}^{6}+\frac{315}{256} C_{1}^{4} C_{2}+\frac{105}{64} C_{1}^{2} C_{2}^{2}+\frac{5}{16} C_{2}^{3}, \quad F_{7}=-\frac{429}{2048} C_{1}^{7}-\frac{693}{512} C_{1}^{5} C_{2}-\frac{315}{128} C_{1}^{3} C_{2}^{2}-\frac{35}{32} C_{1} C_{2}^{3}$,

$F_{8}=\frac{6435}{128} C_{1}^{8}+\frac{3003}{2048} C_{1}^{6} C_{2}+\frac{3465}{1024} C_{1}^{4} C_{2}^{2}+\frac{315}{128} C_{1}^{2} C_{2}^{3}+\frac{35}{128} C_{2}^{4}$.

Higher $F$ 's are of order $J_{2}^{5}$ or smaller.

We now have to evaluate

$$
\int_{-\frac{\pi}{2}}^{\psi} \eta^{n} d \psi=\int_{-\frac{\pi}{2}}^{\psi}(P-Q \sin x)^{n} d x=\int_{0}^{w}(P+Q \cos y)^{n} d y \equiv f_{n}(w),
$$

where

$$
w \equiv \pi / 2+\psi
$$

As in V1961b, p. 178, we now readily show that

$$
f_{n}(w)=\pi^{-1} w f_{n}(\pi)+g_{n}(w)
$$

where $g_{n}(w)$ is an odd function of $w$, of period $2 \pi$. From that same paper

$$
f_{n}(\pi) \equiv \int_{0}^{\pi}(P-Q \cos y)^{n} d y=\pi P^{n}\left(1-e_{1}^{2}\right)^{n / 2} P_{n}\left[\left(1-e_{1}^{2}\right)^{-1 / 2}\right]
$$

where $P_{n}(x)$ is a Legendre polynomial in $x$ and where

$$
\begin{gathered}
e_{1} \equiv Q / P \\
0<e_{1}<1 \text { for } S<0 \\
e_{1}>1 \text { for } S>0 .
\end{gathered}
$$

For $S<0$, the factors $\left(1-e_{1}^{2}\right)^{n / 2}$ and $P_{n}\left[\left(1-e_{1}^{2}\right)^{-1 / 2}\right]$ are both real, for all $n$. For $S>0$, they are both real if $n$ is even and both imaginary if $n$ is odd. In any case their product is real.

If we now introduce, as in V1961b, p. 178,

$$
R_{n}(x) \equiv x^{n} P_{n}(1 / x),
$$

a polynomial of degree $[n / 2]$ in $x^{2}$, we find

$$
\begin{gathered}
\pi^{-1} f_{n}(\pi)=G_{n}(P, Q) \\
G_{n}(P, Q) \equiv P^{n} R_{n}\left(\sqrt{1-e_{1}^{2}}\right),
\end{gathered}
$$

polynomials of degree $n$ in $P$ and $Q$. Through $n=8$ they are given by

$$
G_{0}=1, G_{1}=P, G_{2}=P^{2}+\frac{1}{2} Q^{2}, G_{3}=P^{3}+\frac{3}{2} P Q^{2},
$$




$$
G_{4}=P^{4}+3 P^{2} Q^{2}+\frac{3}{8} Q^{4}, G_{5}=P^{5}+5 P^{3} Q^{2}+\frac{15}{8} P Q^{4},
$$

$$
\begin{aligned}
& 16 G_{6}=16 P^{6}+120 P^{4} Q^{2}+90 P^{2} Q^{4}+5 Q^{6}, \quad 16 G_{7}=16 P^{7}+168 P^{5} Q^{2}+210 P^{3} Q^{4}+35 P Q^{6} \\
& 128 G_{8}=128 P^{8}+1972 P^{6} Q^{2}+3360 P^{4} Q^{4}+1120 P^{2} Q^{6}+35 Q^{8}
\end{aligned}
$$

On inserting (58), (59), and (62) into (56.1), we find

$$
\alpha_{2} u^{-1 / 2} N_{2}=\left(\frac{\pi}{2}+\psi\right) B_{2}+\sum_{n=0}^{\infty} F_{n}\left(C_{1}, C_{2}\right) \sum_{j=1}^{\infty} c_{n j} \cdot \sin j w,
$$

where

$$
B_{2}=\sum_{n=0}^{\infty} F_{n}\left(C_{1}, C_{2}\right) G_{n}(P, Q)
$$

Remembering that $C_{1}, C_{2}$, and $P$ are all of order $J_{2}$ and that $Q$ is of order $J_{2}^{0}$, we have from (64.2), (57), and (63), through order $J_{2}^{4}$ :

$$
\begin{aligned}
B_{2}=1-\frac{1}{2} C_{1} P+\left(\frac{3}{8} C_{1}^{2}+\right. & \left.\frac{1}{2} C_{2}\right)\left(P^{2}+\frac{1}{2} Q^{2}\right)+\frac{9}{64} C_{2}^{2} Q^{4}-\frac{9}{8} C_{1} C_{2} P Q^{2} \\
& -\frac{15}{32} C_{1}^{3} P Q^{2}+\frac{45}{128} C_{1}^{2} C_{2} Q^{4}+\frac{25}{256} C_{2}^{3} Q^{6}+\frac{105}{1024} C_{1}^{4} Q^{4} \\
& +\frac{9}{8} C_{2}^{2} P^{2} Q^{2}-\frac{225}{128} C_{1} C_{2}^{2} P Q^{4}+\frac{525}{1024} C_{1}^{2} C_{2}^{2} Q^{6}+\left(\frac{35}{128}\right)^{2} C_{2}^{4} Q^{8}+O\left(J_{2}^{5}\right) .
\end{aligned}
$$

Note that $B_{2}$ contains $-\frac{1}{2} C_{1} P+\frac{3}{16} C_{1}^{2} Q^{2}$, proportional to $J_{3}^{2} / J_{2}^{2}=O\left(J_{2}^{2}\right)$, so that inclusion of $J_{3}$ in the separable problem apparently leads to new second-order secular terms. We shall say more about this point later.

Let us check $B_{2}$ for $\delta=0$, in which case it should reduce to

$$
B_{2}=\pi^{-1} K(q)=1+\frac{1}{4} q^{2}+\frac{9}{64} q^{4}+\frac{25}{256} q^{6}+\left(\frac{35}{128}\right)^{2} q^{8}+\ldots .
$$

given on p. 184 of V1961b. For $\delta=0$, we have $C_{1}=0, P=0, \eta_{1}=-\eta_{0}, S=\eta_{0}^{2}=\sin ^{2} I$, and $Q=\eta_{0}$ $=\sin I$. For $C_{2}$ we then find from (22.4)

$$
C_{2}=\frac{c^{2}}{a_{0} p_{0}} \frac{p_{0}}{q_{0}} \eta_{0}^{2}=\frac{c^{2}}{a_{0} p_{0}} \frac{\alpha_{2}^{2}}{\alpha_{2}^{2}-\alpha_{3}^{2}} \eta_{0}^{2},
$$

with use of (18) and (21.2). But in the notation of V1961b, $\left(\alpha_{2}^{2}-\alpha_{3}^{2}\right) / \alpha_{2}^{2}=\sin ^{2} i_{0}$, so that we find

$$
\begin{aligned}
C_{2} & =\frac{c^{2}}{a_{0} p_{0}} \frac{\eta_{0}^{2}}{\sin ^{2} i_{0}} \\
& =\eta_{2}^{-2}
\end{aligned}
$$


from p. 173 of V1961b. On inserting these values into (65) and using $q \equiv \eta_{0} / \eta_{2}$, we find

$$
B_{2}=1+\frac{1}{4} q^{2}+\frac{9}{64} q^{4}+\frac{25}{256} q^{6}+\left(\frac{35}{128}\right)^{2} q^{8}+\ldots
$$

This agrees with (66), so that $B_{2}$ reduces correctly for $\delta=0$.

We now have to find the periodic terms in (64.1), i.e., the coefficients $c_{n j}$. Since $F_{5}\left(c_{1}, c_{2}\right)$ is of order $J_{2}^{3}$, we need take $n$ only through the value 4. By expressing $(P+Q \cos y)^{k}, k=0,1,2,3,4$, as trigonometric polynomials, evaluating $\int_{0}^{\omega}(P-Q \cos y)^{k} d y$, picking out the periodic terms, and keeping results only through order $J_{2}^{2}$, we find from (56.1) and (57)

$$
\begin{aligned}
\alpha_{2} u^{-1 / 2} N_{2}=\left(\frac{\pi}{2}+\psi\right) B_{2}+\left(P-\frac{3}{16} C_{1} Q^{2}\right) C_{2} Q \sin w \\
+\frac{1}{32}\left[\left(4 C_{2}+3 C_{1}^{2}\right) Q^{2}+3 C_{2}^{2} Q^{4}\right] \sin 2 w+\frac{C_{1} C_{2}}{16} Q^{3} \sin 3 w \\
+\frac{3}{256} C_{2}^{2} Q^{4} \sin 4 w+\text { periodic terms of order } J_{2}^{3} .
\end{aligned}
$$

In terms of $\psi=w-\pi / 2$, this becomes

$$
\begin{aligned}
\alpha_{2} u^{-1 / 2} N_{2}=\left(\frac{\pi}{2}+\psi\right) B_{2}+ & {\left[C_{2} Q\left(p+\frac{9}{16} C_{1} Q_{2}\right)+\frac{1}{2} C_{1} Q\right] \cos \psi } \\
& -\frac{1}{32}\left[\left(4 C_{2}+3 C_{1}^{2}\right) Q^{2}+3 C_{2}^{2} Q^{4}\right] \sin 2 \psi-\frac{C_{1} C_{2}}{16} Q^{3} \cos 3 \psi \\
& +\frac{3}{256} C_{2}^{2} Q^{4} \sin 4 \psi+\text { periodic terms of } O\left(J_{2}^{3}\right) .
\end{aligned}
$$

For $\delta=0$, we find that the periodic terms in (71) agree with those on p. 184 of V1961b, as they should.

\section{The Integral $N_{1}$}

By (12.1), (50), and (48), we have

$$
\alpha_{2} u^{-1 / 2} N_{1}=\int_{-\frac{\pi}{2}}^{\psi}\left(1+C_{1} \eta-C_{2} \eta^{2}\right)^{-1 / 2} \eta^{2} d \psi .
$$

Then, from (55), (56.2), and (58.1)

$$
\alpha_{2} u^{-1 / 2} N_{1}=\sum_{n=0}^{\infty} F_{n}\left(C_{1}, C_{2}\right) f_{n+2}(w)
$$

As before,

$$
f_{n+2}(w)=w G_{n+2}(P, Q)+g_{n+2}(w),
$$


where $g_{n+2}(w)$ is odd in $w$, with period $2 \pi$. Thus

$$
\alpha_{2} u^{-1 / 2} N_{1}=\left(\frac{\pi}{2}+\psi\right) B_{1}^{\prime}+\sum_{n=0}^{\infty} F_{n}\left(C_{1}, C_{2}\right) g_{n+2}(w)
$$

where

$$
B_{1}^{\prime} \equiv \sum_{n=0}^{\infty} F_{n}\left(C_{1}, C_{2}\right) G_{n+2}(P, Q)
$$

Since the integral $N_{1}$ has a factor $c^{2}=O\left(J_{2}\right)$ in the kinetic equations (11), we need each $F_{n} G_{n+2}$ correct only through order $J_{2}^{3}$, in order to obtain secular terms correct through order $J_{2}^{4}$. From (75.2), (57), and (63), we then find

$$
\begin{aligned}
B_{1}^{\prime}=\frac{1}{2} Q^{2}+P^{2}-\frac{3}{4} C_{1} P Q^{2}+\frac{3}{2} C_{2} P^{2} Q^{2} & +\frac{3}{64}\left(4 C_{2}+3 C_{1}^{2}\right) Q^{4} \\
& -\frac{45}{32} C_{1} C_{2} P Q^{4}+\frac{5}{256}\left(6 C_{2}^{2}+15 C_{1}^{2} C_{2}\right) Q^{6}+\frac{175}{2048} C_{2}^{3} Q^{8}+O\left(J_{2}^{4}\right) .
\end{aligned}
$$

For $\delta=0$, this reduces to

$$
B_{1}^{\prime}=\frac{1}{2} \eta_{0}^{2}+\frac{3}{16} \eta_{2}^{-2} \eta_{0}^{4}+\frac{15}{128} \eta_{2}^{-4} \eta_{0}^{6}+\frac{175}{2048} \eta_{2}^{-6} \eta_{0}^{8}+\ldots
$$

or

$$
B_{1}^{\prime} \eta_{0}^{-2}=\frac{1}{2}+\frac{3}{16} q^{2}+\frac{15}{128} q^{4}+\frac{175}{2048} q^{6}+\ldots
$$

in agreement with the value of $B_{1}$ on p. 184 of V1961b.

To find the periodic terms in (75.1), we proceed just as for $N_{2}$, obtaining

$$
\begin{aligned}
\alpha_{2} u^{-1 / 2} N_{1}=\left(\frac{\pi}{2}+\psi\right) B_{1}^{\prime} & +\left(2 P Q+\frac{3}{8} C_{1} Q^{3}\right) \cos \psi \\
& -\frac{1}{8}\left(2 Q^{2}+C_{2} Q^{4}\right) \sin 2 \psi \\
& +\frac{1}{24} C_{1} Q^{3} \cos 3 \psi \\
& +\frac{1}{64} C_{2} Q^{4} \sin 4 \psi+\text { periodic terms of } O\left(J_{2}^{2}\right) .
\end{aligned}
$$

For $\delta=0$, the periodic terms agree with those appearing in (6.38) of V1961b.

\section{The Integral $N_{3}$}

By (12.3), (50), and (48), we have

$$
\alpha_{2} u^{-1 / 2} N_{3}=\int_{-\frac{\pi}{2}}^{\psi}\left(1-\eta^{2}\right)^{-1}\left(1+C_{1} \eta-C_{2} \eta^{2}\right)^{-1 / 2} d \psi .
$$

Then, from (55) and (56.6),

$$
\alpha_{2} u^{-1 / 2} N_{3}=\sum_{n=0}^{\infty} F_{n}\left(C_{1}, C_{2}\right) L_{n}
$$




$$
L_{n} \equiv \int_{-\frac{\pi}{2}}^{\psi}\left(1-\eta^{2}\right) \eta^{n} d \psi
$$

In particular

$$
L_{0} \equiv \int_{\frac{-\pi}{2}}^{\psi}\left(1-\eta^{2}\right)^{-1} d \psi \quad(81.1) \quad L_{1} \equiv \int_{\frac{\pi}{2}}^{\psi}\left(1-\eta^{2}\right)^{-1} \eta d \psi .
$$

To evaluate the $L_{n}$ s we first prove the lemmas

$$
\begin{array}{cl}
L_{2 \sigma} & =L_{0}-\sum_{\tau=0}^{\sigma-1} \int_{-\frac{\pi}{2}}^{\psi} \eta^{2 \tau} d \psi \quad(\sigma \geqq 1) \\
L_{2 \sigma+1} & =L_{1}-\sum_{\tau=0}^{\sigma-1} \int_{-\frac{\pi}{2}}^{\psi} \eta^{2 \tau+1} d \psi \quad(\sigma \geqq 1) .
\end{array}
$$

To prove them, note first that

$$
\frac{\eta^{n}}{1-\eta}=\frac{1}{1-\eta}-\sum_{k=0}^{n-1} \eta^{k}
$$

and

$$
\frac{\eta^{n}}{1+\eta}=\frac{(-1)^{n}}{1+\eta}-\sum_{k=0}^{n-1}(-1)^{n+k} \eta^{k}
$$

Addition of (83) and (84) then gives

$$
\frac{\eta^{n}}{1-\eta}+\frac{\eta^{n}}{1+\eta}=\frac{1}{1-\eta}+\frac{(-1)^{n}}{1+\eta}-\sum_{k=0}^{n-1}\left[1+(-1)^{n+k}\right] \eta^{k},
$$

reducing to the two cases $n=$ odd and $n=$ even, viz,

$$
\begin{array}{rlrl}
\frac{\eta^{2 \sigma}}{1-\eta^{2}} & =\frac{1}{1-\eta^{2}}-\sum_{\tau=0}^{\sigma-1} \eta^{2 \tau} & & (\sigma \geqq 1) \\
\frac{\eta^{2 \sigma+1}}{1-\eta^{2}} & =\frac{\eta}{1-\eta^{2}}-\sum_{\tau=0}^{\sigma-1} \eta^{2 \tau+1} & (\sigma \geqq 1) .
\end{array}
$$

The lemmas (82) then follow directly from (80.2) and (86).

With application of (58.1), (59), and (62), we then find from them

$$
\begin{array}{rlrl}
L_{2 \sigma} & =L_{0}-w \sum_{\tau=0}^{\sigma-1} G_{2 \tau}(P, Q)-\sum_{\tau=0}^{\sigma-1} g_{2 \tau}(w), & & \sigma \geqq 1 \\
L_{2 \sigma+1} & =L_{1}-w \sum_{\tau=0}^{\sigma-1} G_{2 \tau+1}(P, Q)-\sum_{\tau=0}^{\sigma-1} g_{2 \tau+1}(w), & \sigma \geqq 1,
\end{array}
$$

where $w=\psi+\pi / 2$. Insertion of (87) into (80.1) then gives

$$
\alpha_{2} u^{-1 / 2} N_{3}=L_{0} \sum_{\sigma=0}^{\infty} F_{2 \sigma}+L_{1} \sum_{\sigma=0}^{\infty} F_{2 \sigma+1}+B_{3} w
$$$$
-\sum_{\sigma=1}^{\infty} F_{2 \sigma} \sum_{\tau=0}^{\sigma-1} g_{2 \tau}(w)-\sum_{\sigma=1}^{\infty} F_{2 \sigma+1} \sum_{\tau=0}^{\sigma-1} g_{2 \tau+1}(w),
$$ 


$$
B_{3} \equiv-\sum_{\sigma=1}^{\infty} F_{2 \sigma} \sum_{\tau=0}^{\sigma-1} G_{2 \tau}-\sum_{\sigma=1}^{\infty} F_{2 \sigma+1} \sum_{\tau=0}^{\sigma-1} G_{2 \tau+1}
$$

To simplify the first terms in (88.1), note that by (56.2) and (52), if

$$
h_{0} \equiv i C_{2}^{1 / 2}
$$

the value of $h$ for $\eta=1$, we obtain

$$
F_{2 \sigma}=h_{0}^{2 \sigma} P_{2 \sigma}(\lambda) \quad(90.1) \quad F_{2 \sigma+1}=h_{0}^{2 \sigma+1} P_{2 \sigma+1}(\lambda) .
$$

To evaluate the sums $\sum_{0}^{\infty} F_{2 \sigma}$ and $\sum_{0}^{\infty} F_{2 \sigma+1}$, note that

$$
\left(1-2 \lambda h_{0}+h_{0}^{2}\right)^{-1 / 2}=\sum_{0}^{\infty} h_{0}^{2 \sigma} P_{2 \sigma}(\lambda)+\sum_{0}^{\infty} h_{0}^{2 \sigma+1} P_{2 \sigma+1}(\lambda)
$$

and, since $P_{2 \sigma}(-\lambda)=P_{2 \sigma}(\lambda)$ and $P_{2 \sigma+1}(-\lambda)=-P_{2 \sigma+1}(\lambda)$, that

$$
\left(1+2 \lambda h_{0}+h_{0}^{2}\right)^{-1 / 2}=\sum_{0}^{\infty} h_{0}^{2 \sigma} P_{2 \sigma}(\lambda)-\sum_{0}^{\infty} h_{0}^{2 \sigma+1} P_{2 \sigma+1}(\lambda) .
$$

Solving eqs (91) for each sum, with use of (90), we find

$$
\begin{aligned}
2 \sum_{0}^{\infty} F_{2 \sigma} & =\left(1-2 \lambda h_{0}+h_{0}^{2}\right)^{-1 / 2}+\left(1+2 \lambda h_{0}+h_{0}^{2}\right)^{-1 / 2} \\
2 \sum_{0}^{\infty} F_{2 \sigma+1} & =\left(1-2 \lambda h_{0}+h_{0}^{2}\right)^{-1 / 2}-\left(1+2 \lambda h_{0}+h_{0}^{2}\right)^{-1 / 2} .
\end{aligned}
$$

Since $h=h_{0}$ when $\eta=1$, we have from (53)

$$
1 \mp 2 \lambda h_{0}+h_{0}^{2}=1 \pm C_{1}-C_{2} .
$$

Then from (92) and (93)

$$
\begin{aligned}
L_{0} \sum_{0}^{\infty} F_{2 \sigma}+L_{1} \sum_{0}^{\infty} F_{2 \sigma+1}=\frac{1}{2} L_{0}\left[\left(1+C_{1}-C_{2}\right)^{-1 / 2}+\right. & \left.\left(1-C_{1}-C_{2}\right)^{-1 / 2}\right] \\
& +\frac{1}{2} L_{1}\left[\left(1+C_{1}-C_{2}\right)^{-1 / 2}-\left(1-C_{1}-C_{2}\right)^{-1 / 2}\right] .
\end{aligned}
$$

For $B_{3}$, the coefficient of a secular term, we find from (88.2), (57), and (63), carrying terms through order $J_{2}^{4}$,

$$
\begin{aligned}
B_{3}=-\frac{1}{2} C_{2}-\frac{3}{8} C_{1}^{2}- & \left(\frac{35}{128} C_{1}^{4}+\frac{15}{16} C_{1}^{2} C_{2}+\frac{3}{8} C_{2}^{2}\right)\left(1+\frac{1}{2} Q^{2}\right) \\
& -\frac{3}{8} C_{2}^{2} P^{2}-\left(\frac{105}{64} C_{1}^{2} C_{2}^{2}+\frac{5}{16} C_{2}^{3}\right)\left(1+\frac{1}{2} Q^{2}+\frac{3}{8} Q^{4}\right)
\end{aligned}
$$




$$
\begin{aligned}
-\frac{35}{128} C_{2}^{4}\left(1+\frac{1}{2} Q^{2}+\frac{3}{8} Q^{4}+\frac{5}{16} Q^{6}\right)- & \left(\frac{5}{16} C_{1}^{3}+\frac{3}{4} C_{1} C_{2}\right) P \\
& +\frac{15}{16} C_{1} C_{2}^{2}\left(P+\frac{3}{2} P Q^{2}\right)+O\left(J_{2}^{5}\right) .
\end{aligned}
$$

Here the term $-\frac{3}{8} C_{1}^{2}$ is proportional to $J_{3}^{2} / J_{2}^{2}=O\left(J_{2}^{2}\right)$, so that we apparently again find a secondorder secular term produced by inclusion of $J_{3}$ in the separable problem.

To check (95) for $\delta=0$, put $C_{1}=P=0, C_{2}=\eta_{2}^{-2}$, and $Q=\eta_{0}$. It becomes

$$
\begin{aligned}
B_{3}=\frac{1}{2} \eta_{2}^{-2}+\frac{3}{8} \eta_{2}^{-4}\left(1+\frac{1}{2} \eta_{0}^{2}\right)+\frac{5}{16} \eta_{2}^{-6}(1+ & \left.\frac{1}{2} \eta_{0}^{2}+\frac{3}{8} \eta_{0}^{4}\right) \\
& +\frac{35}{128} \eta_{2}^{-8}\left(1+\frac{1}{2} \eta_{0}^{2}+\frac{3}{8} \eta_{0}^{4}+\frac{5}{16} \eta_{0}^{6}\right)+O\left(J_{2}^{5}\right)
\end{aligned}
$$

agreeing with (6.65) of V1961b through terms of order $J_{2}^{4}$.

To evaluate the periodic terms in (88.1) through order $J_{2}^{2}$, note that all coefficients $F_{n}$ are of order $J_{2}^{3}$ or higher for $n \geqq 5$. Thus we need only $g_{0}, g_{1}$, and $g_{2}$, where $g_{n}$ is the periodic part of

$$
f_{n} \equiv \int_{0}^{w}(P-Q \cos y)^{n} d y .
$$

Then

$$
g_{0}=0, g_{1}=Q \sin w, g_{2}=-2 P Q \sin w+\frac{Q^{2}}{4} \sin 2 w
$$

From (88.1) the relevant periodic terms are then $-F_{3} g_{1}$ and $-F_{4} g_{2}$. With the aid of (96) and (57), their sum then becomes, through order $J_{2}^{2}$,

$$
\begin{aligned}
\text { Periodic terms } & =-\frac{3}{4} C_{1} C_{2} Q \sin w-\frac{3}{32} C_{2}^{2} Q^{2} \sin 2 w \\
& =-\frac{3}{4} C_{1} C_{2} Q \cos \psi+\frac{3}{32} C_{2}^{2} Q^{2} \sin 2 \psi
\end{aligned}
$$

We come next to the evaluation of $L_{0}$ and $L_{1}$. From (81), we have by expansion in partial fractions

$$
\begin{aligned}
& 2 L_{0}=M_{1}^{\prime}+M_{2}^{\prime}, \\
& 2 L_{1}=M_{1}^{\prime}-M_{2}^{\prime},
\end{aligned}
$$

where

$$
M_{1}^{\prime}=\int_{-\pi / 2}^{\psi}(1-\eta)^{-1} d \psi \quad(99.1) \quad M_{2}^{\prime}=\int_{-\pi / 2}^{\psi}(1+\eta)^{-1} d \psi
$$

With the aid of (48) and the definitions

$$
e_{2} \equiv \frac{Q}{1-P} \quad(100.1) \quad e_{3} \equiv \frac{Q}{1+P}
$$


we find

$$
\begin{aligned}
& M_{1}^{\prime}=(1-P)^{-1} \int_{-\pi / 2}^{\psi}\left(1-e_{2} \sin x\right)^{-1} d x \\
& M_{2}^{\prime}=(1+P)^{-1} \int_{-\pi / 2}^{\psi}\left(1+e_{3} \sin x\right)^{-1} d x .
\end{aligned}
$$

Here $e_{2} \leqq 1$ and $e_{3} \leqq 1$, as we shall now prove. Since $|S| \leqq 1$, we have by (31) that $u \approx 1$ and then by $(32.3)$ that

$$
P=\zeta(1-S) \quad(102.1) \quad \zeta=\left(1-\frac{c^{2}}{a_{0} p_{0}} S u\right)^{-1} \frac{\delta}{p_{0}} u
$$

where $0<\zeta<<1$. Now by (47)

$$
(1-P)^{2}-Q^{2}=1-2 P+P^{2}-P^{2}-S=1-2 P-S=(1-S)(1-2 \zeta),
$$

by (102). But $0<\zeta<<1$, so that $1-2 \zeta>0$ and $1-S \geqq 0$, so that $(1-P)^{2}-Q^{2} \geqq 0$ and $1-P \geqq Q$, since neither $1-P$ nor $Q$ is negative. Thus $e_{2} \leqq 1$. Also $(1+P)^{2}-Q^{2}=1+2 P-S \geqq 0$, since $P \geqq 0$ and $S \leqq 1$.

Thus $1+P \geqq Q$ and $e_{3} \leqq 1$.

If in (101.1) we place $x=y-\pi / 2$ and in (101.2) we place $x=y+\pi / 2$, we now find

$$
\begin{aligned}
& M_{1}^{\prime}=(1-P)^{-1} \int_{0}^{\psi+\pi / 2}\left(1+e_{2} \cos y\right)^{-1} d y \\
& M_{2}^{\prime}=(1+P)^{-1} \int_{-\pi}^{\psi-\pi / 2}\left(1+e_{3} \cos y\right)^{-1} d y .
\end{aligned}
$$

To evaluate the $M^{\prime \prime}$ s we define a function $E_{e}^{\prime}(y)$ by

$$
\cos E_{e}^{\prime}=\frac{e+\cos y}{1+e \cos y} \quad \sin E_{e}^{\prime}=\frac{\left(1-e^{2}\right)^{1 / 2} \sin y}{1+e \cos y} \quad(e<1) .
$$

Then when $y=n \pi$, we find $\sin E_{e}^{\prime}=0$ and $\cos E_{e}^{\prime}=(-1)^{n}$. We may therefore impose the further requirement that $E_{e}^{\prime}(y)=y$ whenever $y=n \pi$. The relation between $E_{e}^{\prime}$ and $y$ is then the same as that between the eccentric and true anomalies, respectively, of an ellipse with eccentricity $e$.

We next prove that

$$
\int_{0}^{w}(1+e \cos y)^{-1} d y=\left(1-e^{2}\right)^{-1 / 2} E_{e}^{\prime}(w)
$$

To prove it, we first derive from (104) the relations

$$
\begin{aligned}
& \sin y=\frac{\left(1-e^{2}\right)^{1 / 2} \sin E^{\prime}}{1-e \cos E^{\prime}} \\
& \frac{1+e^{2}}{1+e \cos y}=1-e \cos E^{\prime} .
\end{aligned}
$$


Differentiation of (107) gives

$$
\frac{\left(1-e^{2}\right) \sin y d y}{(1+e \cos y)^{2}}=\sin E^{\prime} d E^{\prime}
$$

Division of (108) by (107) then shows that

$$
\frac{\sin y d y}{1+e \cos y}=\frac{\sin E^{\prime} d E^{\prime}}{1-e \cos E^{\prime}}=\left(1-e^{2}\right)^{-1 / 2} \sin y d E^{\prime},
$$

by (106). Thus

$$
\frac{d y}{1+e \cos y}=\left(1-e^{2}\right)^{-1 / 2} d E^{\prime}
$$

from which theorem (105) follows at once, since $E^{\prime}=0$ when $y=0$. Then, since $E_{e}^{\prime}(0)=0$ and $E_{e}^{\prime}(-\pi)=-\pi$, we find on applying (105) to (103),

$$
\begin{aligned}
& M_{1}^{\prime}=(1-P)^{-1}\left(1-e_{2}^{2}\right)^{-1 / 2} E_{2}^{\prime}(\psi+\pi / 2) \\
& M_{2}^{\prime}=(1+P)^{-1}\left(1-e_{3}^{2}\right)^{-1 / 2}\left[E_{3}^{\prime}(\psi-\pi / 2)+\pi\right] .
\end{aligned}
$$

From (111), (100), and $Q^{2}=P^{2}+S$, it then follows that

$$
\begin{aligned}
& M_{1}^{\prime}=(1-S-2 P)^{-1 / 2} E_{2}^{\prime}(\psi+\pi / 2) \\
& M_{2}^{\prime}=(1-S+2 P)^{-1 / 2}\left[E_{3}^{\prime}(\psi-\pi / 2)+\pi\right] .
\end{aligned}
$$

By (112), (98), and (102), we then find

$$
\begin{aligned}
& (1-S)^{1 / 2} L_{0}=\frac{\pi / 2}{\sqrt{1+2 \zeta}}+\chi_{0} \\
& (1-S)^{1 / 2} L_{1}=\frac{-\pi / 2}{\sqrt{1+2 \zeta}}+\chi_{1},
\end{aligned}
$$

where

$$
\begin{aligned}
& 2 \chi_{0}=\frac{E_{2}^{\prime}(\psi+\pi / 2)}{\sqrt{1-2 \zeta}}+\frac{E_{3}^{\prime}(\psi-\pi / 2)}{\sqrt{1+2 \zeta}} \\
& 2 \chi_{1}=\frac{E_{2}^{\prime}(\psi+\pi / 2)}{\sqrt{1-2 \zeta}}-\frac{E_{3}^{\prime}(\psi-\pi / 2)}{\sqrt{1+2 \zeta}} .
\end{aligned}
$$

From (88.1), (94), (97), and (113), there follows the complete expression for $N_{3}$

$$
\begin{aligned}
\alpha_{2} u^{-1 / 2} N_{3}=\frac{1}{2}[(1+ & \left.\left.C_{1}-C_{2}\right)^{-1 / 2}+\left(1-C_{1}-C_{2}\right)^{-1 / 2}\right](1-S)^{-1 / 2}\left[\frac{\pi / 2}{\sqrt{1+2 \zeta}}+\chi_{0}\right] \\
& +\frac{1}{2}\left[\left(1+C_{1}-C_{2}\right)^{-1 / 2}-\left(1-C_{1}-C_{2}\right)^{-1 / 2}\right](1-S)^{-1 / 2}\left[\frac{-\pi / 2}{\sqrt{1+2 \zeta}}+\chi_{1}\right] \\
& +(\psi+\pi / 2) B_{3}-\frac{3}{4} C_{1} C_{2} Q \cos \psi+\frac{3}{32} C_{2}^{2} Q^{2} \sin 2 \psi+\text { periodic terms of } O\left(J_{2}^{3}\right) .
\end{aligned}
$$


Here $B_{3}$ is given by (95) and $\chi_{0}$ and $\chi_{1}$ are given by (114).

To check this expression for $\delta=0$, note that then $C_{1}=0, \zeta=0, S=\eta_{0}^{2}, u=\left(\alpha_{2}^{2}-\alpha_{3}^{2}\right)^{-1} \alpha_{2}^{2} \eta_{0}^{2}$, so that $\alpha_{2} u^{-1 / 2}$ becomes $\left(\alpha_{2}^{2}-\alpha_{3}^{2}\right)^{1 / 2} \eta_{0}^{-1}$. We have to compare with eq (6.64) of V1961b. Now we have already checked $B_{3}$ and the periodic terms in (115). The check will thus consist only in showing that the terms involving $\chi_{0}$ and $\chi_{1}$ in (115) reduce to the expression $\left(1-\eta_{0}^{2}\right)^{-1 / 2}\left(1-\eta_{2}^{-2}\right)^{-1 / 2} \chi$ of V1961b.

Since $C_{1}=0$ for $\delta=0$, the term involving $\chi_{1}$ disappears, because of the minus sign inside the brackets. Since $C_{2}$ reduces to $\eta_{2}^{-2}$, the first line becomes

$$
\left(1-\eta_{0}^{2}\right)^{-1 / 2}\left(1-\eta_{2}^{-2}\right)^{-1 / 2}\left(\frac{\pi}{2}+\chi_{0}\right)
$$

Now the $\pi / 2$ is an additive constant, arising only because we now have the lower limit $\eta_{1}$ in the $\eta$-integrals instead of zero. We shall later get rid of it on replacing the constant $\beta_{2}^{\prime}$ by another constant $\beta_{2}$. Thus we now have only to verify that $\chi_{0}$ reduces to $\chi$ when $\delta=0$. When $\delta=0$, $e_{2}=(1-P)^{-1} Q$ reduces to $\eta_{0}$, and we find from (114.1) and (104), with $\zeta=0$, that

$$
\cos 2 \chi_{0}=\frac{\cos ^{2} \psi-\left(1-\eta_{0}^{2}\right) \sin ^{2} \psi}{1-\eta_{0}^{2} \sin ^{2} \psi} \quad \sin 2 \chi_{0}=\frac{2 \sqrt{1-\eta_{0}^{2}} \sin \psi \cos \psi}{1-\eta_{0}^{2} \sin ^{2} \psi}
$$

But the $\chi$ of V1961b, by p. 516 of V1961c, satisfies

$$
\cos \chi=\frac{\cos \psi}{\sqrt{1-\eta_{0}^{2} \sin ^{2} \psi}} \quad \sin \chi=\frac{\sqrt{1-\eta_{0}^{2}} \sin \psi}{\sqrt{1-\eta_{0}^{2} \sin ^{2} \psi}} .
$$

One verifies easily that $\cos 2 \chi_{0}=\cos 2 \chi$ and $\sin 2 \chi_{0}=\sin 2 \chi$. Thus in the limit $\delta=0$, the quantity $\chi_{0}$ and thus the integral $N_{3}$ both reduce correctly.

\section{Assembly of Equations for the Inversion}

From (11), (67), (78), and (115) of the present paper and (5.30), (5.35), and (5.60) of V1961b, we have

$t+\beta_{1}=\left(-2 \alpha_{1}\right)^{-\mathbf{1} / 2}\left[b_{1} E+a(E-e \sin E)+A_{1} v+A_{11} \sin v+A_{12} \sin 2 v\right]$

$$
+c^{2} \alpha_{2}^{-1} u^{1 / 2}\left[\left(\psi+\frac{\pi}{2}\right) B_{1}^{\prime}+B_{11} \cos \psi+B_{12} \sin 2 \psi+B_{13} \cos 3 \psi+B_{14} \sin 4 \psi\right]
$$

$\beta_{2}^{\prime} \alpha_{2}^{-1}=-\left(-2 \alpha_{1}\right)^{-1 / 2}\left[A_{2} v+\sum_{k=1}^{4} A_{2 k} \sin k v\right]+\alpha_{2}^{-1} u^{1 / 2}\left[\left(\psi+\frac{\pi}{2}\right) B_{2}+B_{21} \cos \psi\right.$

$$
\left.+B_{22} \sin 2 \psi+B_{23} \cos 3 \psi+B_{24} \sin 4 \psi\right]
$$

where

$$
\begin{aligned}
B_{11} & =2 P Q-\frac{3}{8} C_{1} Q^{3}, & B_{12} & =-\left(\frac{Q^{2}}{4}+\frac{1}{8} C_{2} Q^{4}\right) \\
B_{13} & =-C_{1} Q^{3} / 24, & B_{14} & =C_{2} Q^{4} / 64
\end{aligned}
$$




$$
\begin{gathered}
B_{21}=C_{2} P Q-\frac{9}{16} C_{1} C_{2} Q^{3}+\frac{1}{2} C_{1} Q, \quad B_{22}=-\frac{1}{32}\left[\left(4 C_{2}+3 C_{1}^{2}\right) Q^{2}+3 C_{2}^{2} Q^{4}\right] \\
B_{23}=-\frac{1}{16} C_{1} C_{2} Q^{3}, \quad B_{24}=\frac{3}{256} C_{2}^{2} Q^{4}
\end{gathered}
$$

and where $B_{2}^{\prime}$ and $B_{2}$ are given by (76) and (65).

Note that by V1962 the restrictions on p. 176 of V196lb, concerning orbital inclinations, have been removed.

Before proceeding further, it is convenient to eliminate the terms above involving $\pi / 2$. If we lump the term $-c^{2} \alpha_{2}^{-1} u^{1 / 2} B_{1}^{\prime} \pi / 2$ into $\beta_{1}$, the physical meaning of $\beta_{1}$ will not be essentially changed. In the limit $J_{2} \rightarrow 0$, this change will vanish, since $c^{2}=O\left(J_{2}\right)$, and $-\beta_{1}$ will still reduce in the elliptic limit to $\tau$, the time of passage through perigee. Also, for $J_{2}=0$ and $J_{3}=0, u=1$ and $B_{2}=1$, so that the term $-\alpha_{2}^{-1} u^{1 / 2} B_{2} \pi / 2$ reduces to $-\frac{1}{2} \pi \alpha_{2}^{-1}$. Since $\beta_{2}^{\prime} \rightarrow \omega+\pi / 2$ in the elliptic limit, if we put

$$
\beta_{2}=\beta_{2}^{\prime}-u^{1 / 2} B_{2} \pi / 2
$$

the new constant $\beta_{2}$ will reduce in the elliptic limit to $\omega$, the argument of perigee

With these changes, we then have

$$
\begin{aligned}
t+\beta_{1}=\left(-2 \alpha_{1}\right)^{-1 / 2}\left[b_{1} E+\right. & \left.a(E-e \sin E)+A_{1} v+A_{11} \sin v+A_{12} \sin 2 v\right] \\
& +c^{2} \alpha_{2}^{-1} u^{1 / 2}\left[B_{1}^{\prime} \psi+B_{11} \cos \psi+B_{12} \sin 2 \psi+B_{13} \cos 3 \psi+B_{14} \sin 4 \psi\right]
\end{aligned}
$$

$$
\begin{aligned}
\beta_{22} \alpha_{2}^{-1}=-(-2 \alpha)^{-1 / 2}\left[A_{2} v+\right. & \left.\sum_{k=1}^{4} A_{2 k} \sin k v\right] \\
& +\alpha_{22}^{-1} u^{1 / 2}\left[B_{22} \psi+B_{21} \cos \psi+B_{22} \sin 2 \psi+B_{23} \cos 3 \psi+B_{24} \sin 4 \psi\right] .
\end{aligned}
$$

To carry out the inversion, we write

$$
E=M_{s}+E_{p}, \quad v=M_{s}+E_{p}, \quad \psi=\psi_{s}+\psi_{p},
$$

where the subscript $s$ means "secular" and the subscript $p$ "periodic."

\section{The Secular Solution}

To obtain the secular terms $M_{s}$ and $\psi_{s}$, we put $E=v=M_{s}$ in (118) and discard all the sines and cosines, thereby obtaining for $M_{s}$ and $\psi_{s}$ the pair of linear algebraic equations:

$$
\begin{gathered}
\left(-2 \alpha_{1}\right)^{-1 / 2}\left(a+b_{1}+A_{1}\right) M_{s}+c^{2} \alpha_{2}^{-1} u^{1 / 2} B_{1}^{\prime} \psi_{s}=t+\beta_{1} \\
-\left(-2 \alpha_{1}\right)^{1 / 2} A_{2} M_{s}+\alpha_{2}^{-1 / 2} u^{1 / 2} B_{2} \psi_{s}=\beta_{2} \alpha_{2}^{-1} .
\end{gathered}
$$

Their solution is

$$
M_{s}=l_{0}+2 \pi \nu_{1} t \quad(121.1), \quad \psi_{s}=l_{0}+g_{0}+2 \pi \nu_{2} t
$$

where

$$
2 \pi \nu_{1}=\left(-2 \alpha_{1}\right)^{1 / 2}\left(a+b_{1}+A_{1}+c^{2} A_{2} B_{1}^{\prime} B_{2}^{-1}\right)^{-1}
$$




$$
\begin{gathered}
2 \pi \nu_{2}=\alpha_{2} u^{-1 / 2} A_{2} B_{2}^{-1}\left(a+b_{1}+A_{1}+c^{2} A_{2} B_{1}^{\prime} B_{2}^{-1}\right)^{-1} \\
l_{0}=2 \pi \nu_{1}\left(\beta_{1}-c^{2} \beta_{2} \alpha_{2}^{-1} B_{1}^{\prime} B_{2}^{-1}\right) \\
l_{0}+g_{0}=2 \pi \nu_{2}\left[\beta_{1}+\beta_{2} \alpha_{2}^{-1}\left(a+b_{1}+A_{1}\right) A_{2}^{-1}\right] .
\end{gathered}
$$

\section{The Fundamental Frequencies}

By V1961a and pp. 188 and 189 of V1961b, the above $\nu$ 's should satisfy

$$
\nu_{1}=\frac{\partial \alpha_{1}}{\partial j_{1}} \quad(124.1) \quad \nu_{2}=\frac{\partial \alpha_{1}}{\partial j_{2}}
$$

where $j_{1}$ and $j_{2}$ are the action variables corresponding to $\rho$ and $\eta$, respectively. From V196lb, they should thus satisfy

$$
\nu_{1}=\left(j_{11} j_{22}-j_{12} j_{21}\right)^{-1} j_{22}, \quad \nu_{2}=-\left(j_{11} j_{22}-j_{12} j_{21}\right)^{-1} j_{12}
$$

where

$$
\begin{gathered}
j_{11}=2 \pi\left(-2 \alpha_{1}\right)^{-1 / 2}\left(a+b_{1}+A_{1}\right), \quad j_{12}=-2 \pi \alpha_{2}\left(-2 \alpha_{1}\right)^{-1 / 2} A_{2} \\
j_{21}=2 c^{2} N_{1}\left(\eta_{0}\right), \quad j_{22}=2 \alpha_{2} N_{2}\left(\eta_{0}\right) .
\end{gathered}
$$

Now when $\eta=\eta_{0}$, we have $\psi=\pi / 2$, by (45.1), (47), and (48). Then, by (71), (78), and (127)

$$
j_{21}=2 \pi c^{2} \alpha_{2}^{-1} u^{1 / 2} B_{1}^{\prime}, \quad j_{22}=2 \pi u^{1 / 2} B_{2} .
$$

By means of (125) through (128), we readily verify that the fundamental frequencies $\partial \alpha_{1} / \partial j_{1}$ and $\partial \alpha_{2} / \partial j_{2}$ agree with the expressions given in (122).

\section{Periodic Terms}

In calculating the periodic terms through the second order, we first set

$$
E_{p}=E_{0}+E_{1}+E_{2}, \quad v_{p}=v_{0}+v_{1}+v_{2}, \quad \psi_{p}=\psi_{0}+\psi_{1}+\psi_{2},
$$

where the subscript denotes the order of a term in $J_{2}$. We shall calculate the zeroth, first, and second order terms in succession.

Before we can do so, however, we need a table of the order of the various coefficients and constant quantities. Including the quantities necessary for the right ascension $\varphi$ also, we find

\begin{tabular}{c|c||c|c}
\hline \hline Quantity & Order & Quantity & Order \\
\hline$A_{2}$ & $J_{2}^{0}$ & $B_{3}$ & $J_{2}$ \\
$B_{1}^{\prime}$ & $J_{2}^{0}$ & $A_{11}$ & $J_{2}^{2}$ \\
$B_{2}$ & $J_{2}^{0}$ & $A_{12}$ & $J_{2}^{2}$ \\
$B_{12}$ & $J_{2}^{0}$ & $A_{23}$ & $J_{2}^{2}$ \\
$b_{1}$ & $J_{2}$ & $A_{24}$ & $J_{2}^{2}$ \\
$A_{1}$ & $J_{2}$ & $B_{21}$ & $J_{2}^{2}$ \\
$B_{11}$ & $J_{2}$ & $B_{23}^{\prime}$ & $J_{2}^{2}$ \\
$A_{21}$ & $J_{2}$ & $B_{24}$ & $J_{2}^{2}$ \\
$A_{22}$ & $J_{2}$ & & \\
$B_{13}$ & $J_{2}$ & & \\
$B_{14}$ & $J_{2}$ & & \\
$B_{22}$ & $J_{2}$ & & \\
\hline
\end{tabular}




\subsection{Zeroth Order Periodic Terms}

To find $E_{0}, v_{0}$, and $\psi_{0}$, place $E=M_{s}+E_{0}, v=M_{s}+v_{0}, \psi=\psi_{s}+\psi_{0}$ in (118), retain secular terms with full accuracy, and discard all periodic quantities of order higher than $J_{2}^{0}$. However, as cautioned in V1961b, keep all terms that are retained to as high an accuracy as possible; such a procedure simplifies the resulting formulas. Then

$$
\begin{gathered}
t+\beta_{1}=\left(-2 \alpha_{1}\right)^{-1 / 2}\left[\left(a+b_{1}\right)\left(M_{s}+E_{0}\right)-a e \sin \left(M_{s}+E_{0}\right)+A_{1} M_{s}\right]+c^{2} \alpha_{2}^{-1} u^{1 / 2} B_{1}^{\prime} \psi_{s} \\
\beta_{2} \alpha_{2}^{-1}=-\left(2 \alpha_{1}\right)^{-1 / 2} A_{2}\left(M_{s}+v_{0}\right)+\alpha_{2}^{-1} u^{1 / 2} B_{2}\left(\psi_{s}+\psi_{0}\right) .
\end{gathered}
$$

Subtraction of (120.1) from (129.1) then gives

$$
\left(a+b_{1}\right) E_{0}-a e \sin \left(M_{s}+E_{0}\right)=0 .
$$

On dividing (120) by $a+b_{1}$ and adding $M_{s}$ to both sides of the result, we find a Kepler equation for $M_{s}+E_{0}$, viz,

$$
\begin{gathered}
M_{s}+E_{0}-e^{\prime} \sin \left(M_{s}+E_{0}\right)=M_{s} \\
e^{\prime} \equiv \frac{a e}{a+b_{1}}<e<1 .
\end{gathered}
$$

Solve this Kepler equation for $E=M_{s}+E_{0}$. Then find $v=M_{s}+v_{0}$ by the anomaly connections

$$
\cos v=\frac{\cos E-e}{1-e \cos E} \quad \sin v=\frac{\left(1-e^{2}\right)^{1 / 2} \sin E}{1-e \cos E}
$$

To find $\psi_{0}$, subtract (120.2) from (129.2) and solve for $\psi_{0}$. The result is

$$
\psi_{0}=\alpha_{2}\left(-2 \alpha_{1}\right)^{-1 / 2} u^{-1 / 2} A_{2} B_{2}^{-1} v_{0} .
$$

As in V1961b, this gives $\psi_{0}=v_{0}+O\left(J_{2}\right)$, but it is important to retain the coefficient with full accuracy, for later simplifications.

\subsection{First Order Periodic Terms}

We now place $E=M_{s}+E_{0}+E_{1}, v=M_{s}+v_{0}+v_{1}, \psi=\psi_{s}+\psi_{0}+\psi_{1}$ in (118), discarding periodic terms of order $J_{2}^{2}$, and use our knowledge of $M_{s}, \psi_{s}, E_{0}, v_{0}$, and $\psi_{0}$ to find $E_{1}, v_{1}$, and $\psi_{1}$. From (118) we obtain

$$
\begin{aligned}
t+\beta_{1}=\left(-2 \alpha_{1}\right)^{-1 / 2}\left[\left(a+b_{1}\right)\left(M_{s}+E_{0}+E_{1}\right)-a e \sin \left(M_{s}+E_{0}+E_{1}\right)\right. & \\
& \left.+A_{1}\left(M_{s}+v_{0}\right)\right]+c^{2} \alpha_{2}^{-1} u^{1 / 2}\left[B_{1}^{\prime}\left(\psi_{s}+\psi_{0}\right)+B_{12} \sin \left(2 \psi_{s}+2 \psi_{0}\right)\right] \\
\beta_{2} \alpha_{2}^{-1}=-\left(-2 \alpha_{1}\right)^{-1 / 2}\left[A_{2}\left(M_{s}+v_{0}+v_{1}\right)\right. & \left.+A_{21} \sin \left(M_{s}+v_{0}\right)+A_{22} \sin \left(2 M_{s}+2 v_{0}\right)\right] \\
& +\alpha_{2}^{-1} u^{1 / 2}\left[B_{2}\left(\psi_{s}+\psi_{0}+\psi_{1}\right)+B_{222} \sin \left(2 \psi_{s}+2 \psi_{0}\right)\right] .
\end{aligned}
$$


On subtracting (120.1) from (134.1), transposing some terms, and dividing through by $\left(-2 \alpha_{1}\right)^{-1 / 2}$ $\left(a+b_{1}\right)$, we find:

$$
\begin{aligned}
E_{0}+E_{1}-e^{\prime} \sin \left(M_{s}+E_{0}+E_{1}\right)=-\left(a+b_{1}\right)^{-1}\left[A_{1} v_{0}+c^{2} \alpha_{2}^{-1}\left(-2 \alpha_{1}\right)^{1 / 2} u^{1 / 2} B_{1}^{\prime} \psi_{0}\right. & \\
& \left.+c^{2} \alpha_{2}^{-1}\left(-2 \alpha_{1}\right)^{1 / 2} u^{1 / 2} B_{12} \sin \left(2 \psi_{s}+2 \psi_{0}\right)\right],
\end{aligned}
$$

where $e^{\prime}$ is given by (131.2). By (133) the terms in $v_{0}$ and $\psi_{0}$ consolidate into $\left(A_{1}+c^{2} A_{2} B_{1}^{\prime} B_{2}^{-1}\right) v_{0}$ and (135) becomes

$$
M_{s}+E_{0}+E_{1}-e^{\prime} \sin \left(M_{s}+E_{0}+E_{1}\right)=M_{s}+M_{1},
$$

where

$$
M_{1}=-\left(a+b_{1}\right)^{-1}\left[\left(A_{1}+c^{2} A_{2} B_{1}^{\prime} B_{2}^{-1}\right) v_{0}+c^{2} \alpha_{2}^{-1}\left(-2 \alpha_{1}\right)^{1 / 2} u^{1 / 2} B_{12} \sin \left(2 \psi_{s}+2 \psi_{0}\right)\right] .
$$

By p. 194 of V1961b, the solution for $E_{1}$ is then given by

$$
E_{1}=\frac{M_{1}}{1-e^{\prime} \cos \left(M_{s}+E_{0}\right)}-\frac{e^{\prime}}{2} \frac{M_{1}^{2} \sin \left(M_{s}+E_{0}\right)}{\left[1-e^{\prime} \cos \left(M_{s}+E_{0}\right)\right]^{3}}+O\left(J_{2}^{3}\right) \cdot
$$

To find $v_{1}$, insert the known values of $E=M_{s}+E_{0}+E_{1}$ and $e$ into the anomaly connections (132) and solve for $v=M_{s}+v_{0}+v_{1}$.

To find $\psi_{1}$, subtract (120.2) from (134.2). The result is

$$
\begin{aligned}
\alpha_{2}^{-1} u^{1 / 2}\left[B_{2}\left(\psi_{0}+\psi_{1}\right)+B_{22} \sin \left(2 \psi_{s}+2 \psi_{0}\right)\right]=\left(-2 \alpha_{1}\right)^{-1 / 2}\left[A_{2}\left(v_{0}+v_{1}\right)\right. & \\
& \left.+A_{21} \sin \left(M_{s}+v_{0}\right)+A_{22} \sin \left(2 M_{s}+2 v_{0}\right)\right] .
\end{aligned}
$$

By virtue of (133) the terms proportional to $\psi_{0}$ and $v_{0}$ cancel out. On solving for $\psi_{1}$, we then find $\psi_{1}=-B_{22} B_{2}^{-1} \sin \left(2 \psi_{s}+2 \psi_{0}\right)+\alpha_{2}\left(-2 \alpha_{1}\right)^{-1 / 2} u^{-1 / 2} B_{2}^{-1}\left[A_{2} v_{1}\right.$

$$
\left.+A_{21} \sin \left(M_{s}+v_{0}\right)+A_{22} \sin \left(2 M_{s}+2 v_{0}\right)\right] .
$$

\subsection{Second Order Periodic Terms}

To find $E_{2}, v_{2}$, and $\psi_{2}$, insert $E=M_{s}+E_{0}+E_{1}+E_{2}, v=M_{s}+v_{0}+v_{1}+v_{2}$, and $\psi=\psi_{s}+\psi_{0}+\psi_{1}+\psi_{2}$ into (118), retaining all periodic terms of order $J_{2}^{2}$ and discarding all those of higher order. We obtain

$t+\beta_{1}=\left(-2 \alpha_{1}\right)^{-1 / 2}\left[\left(a+b_{1}\right)\left(M_{s}+E_{0}+E_{1}+E_{2}\right)-a e \sin \left(M_{s}+E_{0}+E_{1}+E_{2}\right)\right.$

$$
\begin{gathered}
\left.+A_{1}\left(M_{s}+v_{0}+v_{1}\right)+A_{11} \sin \left(M_{s}+v_{0}\right)+A_{12} \sin \left(2 M_{s}+2 v_{0}\right)\right] \\
+c^{2} \alpha_{2}^{-1} u^{1 / 2}\left[B_{1}^{\prime}\left(\psi_{s}+\psi_{0}+\psi_{1}\right)+B_{11} \cos \left(\psi_{s}+\psi_{0}\right)+B_{12} \sin \left(2 \psi_{s}+2 \psi_{0}+2 \psi_{1}\right)\right. \\
\left.+B_{13} \cos \left(3 \psi_{s}+3 \psi_{0}\right)+B_{14} \sin \left(4 \psi_{s}+4 \psi_{0}\right)\right] .
\end{gathered}
$$




$$
\begin{gathered}
\beta_{2} \alpha_{22}^{-1}=-\left(-2 \alpha_{1}\right)^{-1 / 2}\left[A_{2}\left(M_{s}+v_{0}+v_{1}+v_{2}\right)+A_{21} \sin \left(M_{s}+v_{0}+v_{1}\right)+A_{22} \sin \left(2 M_{s}+2 v_{0}+2 v_{1}\right)\right. \\
\left.+A_{23} \sin \left(3 M_{s}+3 v_{0}\right)+A_{24} \sin \left(4 M_{s}+4 v_{0}\right)\right]+\alpha_{2}^{-1} u^{1 / 2}\left[B_{2}\left(\psi_{s}+\psi_{0}+\psi_{1}+\psi_{2}\right)+B_{21} \cos \left(\psi_{s}+\psi_{0}\right)\right. \\
\left.+B_{222} \sin \left(2 \psi_{s}+2 \psi_{0}+2 \psi_{1}\right)+B_{23} \cos \left(3 \psi_{s}+3 \psi_{0}\right)+B_{24} \sin \left(4 \psi_{s}+4 \psi_{0}\right)\right] .
\end{gathered}
$$

To solve for $E_{2}$, subtract (134.1) from (140.1). We find

$$
\begin{array}{r}
\left(-2 \alpha_{1}\right)^{-1 / 2}\left[\left(a+b_{1}\right) E_{2}-a e \sin \left(M_{s}+E_{0}+E_{1}+E_{2}\right)+a e \sin \left(M_{s}+E_{0}+E_{1}\right)+A_{1} v_{1}+A_{11} \sin \left(M_{s}+v_{0}\right)\right. \\
\left.+A_{12} \sin \left(2 M_{s}+2 v_{0}\right)\right]+c^{2} \alpha_{2}^{-1} u^{1 / 2}\left[B_{1}^{\prime} \psi_{1}+B_{11} \cos \left(\psi_{s}+\psi_{0}\right)+B_{12} \sin \left(2 \psi_{s}+2 \psi_{0}+2 \psi_{1}\right)\right. \\
\left.-B_{12} \sin \left(2 \psi_{s}+2 \psi_{0}\right)+B_{13} \cos \left(3 \psi_{s}+3 \psi_{0}\right)+B_{14} \sin \left(4 \psi_{s}+4 \psi_{0}\right)\right] .
\end{array}
$$

Now

$$
\sin \left(M_{s}+E_{0}+E_{1}+E_{2}\right)-\sin \left(M_{s}+E_{0}+E_{1}\right)=E_{2} \cos \left(M_{s}+E_{0}+E_{1}\right)+O\left(J_{2}^{4}\right)
$$

and

$$
c^{2} \sin \left(2 \psi_{s}+2 \psi_{0}+2 \psi_{1}\right)-c^{2} \sin \left(2 \psi_{s}+2 \psi_{0}\right)=2 c^{2} \psi_{1} \cos \left(2 \psi_{s}+2 \psi_{0}\right)+O\left(J_{2}^{3}\right),
$$

where $E_{2}$ and $\psi_{1}$ are in radians. On inserting (142) into (141) and solving for $E_{2}$, we find

$$
\begin{gathered}
E_{2}=\frac{M_{2}}{1-e^{\prime} \cos \left(M_{s}+E_{0}+E_{1}\right)} \\
M_{22} \equiv-\left(a+b_{1}\right)^{-1} \mid A_{1} v_{1}+A_{11} \sin \left(M_{s}+v_{0}\right)+A_{12} \sin \left(2 M_{s}+2 v_{0}\right) \\
+c^{2} \alpha_{2}^{-1}\left(-2 \alpha_{1}\right)^{1 / 2} u^{1 / 2}\left\{B_{1}^{\prime} \psi_{1}+B_{11} \cos \left(\psi_{s}+\psi_{0}\right)+2 B_{12} \psi_{1} \cos \left(2 \psi_{s}+2 \psi_{0}\right)\right. \\
\left.\left.+B_{13} \cos \left(3 \psi_{s}+3 \psi_{0}\right)+B_{14} \sin \left(4 \psi_{s}+4 \psi_{0}\right)\right\}\right] .
\end{gathered}
$$

Comparison with (8.45) of V1961b shows that a term in $\sin \left(2 \psi_{s}+2 \psi_{0}\right)$ is now missing. The reason is that, by using the full accuracy for $B_{12}$ in the first order periodic terms, we have already included it in $M_{1}$.

To find $v_{2}$, insert $E=M_{s}+E_{0}+E_{1}+E_{2}$ in the anomaly connections (132) and solve for $v$ $=M_{s}+v_{0}+v_{1}+v_{2}$.

To find $\psi \cdot 2$, subtract (134.2) from (140.2) to obtain

$$
\begin{aligned}
-\left(-2 \alpha_{1}\right)^{-1 / 2}\left[A_{22} v_{2}+\right. & A_{21} \sin \left(M_{s}+v_{0}+v_{1}\right)-A_{21} \sin \left(M_{s}+v_{0}\right) \\
& +A_{222} \sin \left(2 M_{s}+2 v_{0}+2 v_{1}\right)-A_{222} \sin \left(2 M_{s}+2 v_{0}\right) \\
& \left.+A_{223} \sin \left(3 M_{s}+3 v_{0}\right)+A_{24} \sin \left(4 M_{s}+4 v_{0}\right)\right] \\
& +\alpha_{22}^{-1} u^{1 / 2}\left[B_{22} \psi_{22}+B_{21} \cos \left(\psi_{s}+\psi_{0}\right)+B_{22} \sin \left(2 \psi_{s}+2 \psi_{0}+2 \psi_{1}\right)\right. \\
& \left.-B_{222} \sin \left(2 \psi_{s}+2 \psi_{0}\right)+B_{23} \cos \left(3 \psi_{s}+3 \psi_{0}\right)+B_{24} \cos \left(4 \psi_{s}+4 \psi_{0}\right)\right]=0 .
\end{aligned}
$$


On inserting

$$
A_{21} \sin \left(M_{s}+v_{0}+v_{1}\right)-A_{21} \sin \left(M_{s}+v_{0}\right)=A_{21} v_{1} \cos \left(M_{s}+v_{0}\right)+O\left(J_{2}^{3}\right)
$$

$$
\begin{aligned}
& A_{22} \sin \left(2 M_{s}+2 v_{0}+2 v_{1}\right)-A_{22} \sin \left(2 M_{s}+2 v_{0}\right)=2 A_{22} v_{1} \cos \left(2 M_{s}+2 v_{0}\right)+O\left(J_{2}^{3}\right) \\
& B_{22} \sin \left(2 \psi_{s}+2 \psi_{0}+2 \psi_{1}\right)-B_{22} \sin \left(2 \psi_{s}+2 \psi_{0}\right)=2 B_{22} \psi_{1} \cos \left(2 \psi_{s}+2 \psi_{0}\right)+O\left(J_{2}^{3}\right)
\end{aligned}
$$

into (144) and solving for $\psi_{2}$, we find

$\psi_{2}=-B_{2}^{-1}\left[B_{21} \cos \left(\psi_{s}+\psi_{0}\right)+2 B_{22} \psi_{1} \cos \left(2 \psi_{s}+2 \psi_{0}\right)+B_{23} \cos \left(3 \psi_{s}+3 \psi_{0}\right)+B_{24} \sin \left(4 \psi_{s}+4 \psi_{0}\right)\right]$

$$
\begin{array}{r}
+\alpha_{2} u^{-1 / 2}\left(-2 \alpha_{1}\right)^{-1 / 2} B_{2}^{-1}\left[A_{22} v_{2}+A_{21} v_{1} \cos \left(M_{s}+v_{0}\right)+2 A_{22} v_{1} \cos \left(2 M_{s}+2 v_{0}\right)\right. \\
\left.+A_{23} \sin \left(3 M_{s}+3 v_{0}\right)+A_{24} \sin \left(4 M_{s}+4 v_{0}\right)\right] .
\end{array}
$$

This completes the solution for the $E, v$, and $\psi$ and thus for the spheroidal coordinates $\rho$ and $\eta$.

\section{The Right Ascension}

To find $\varphi$ we have to insert (115) for $N_{3}$ and (5.60) of V1961b for $R_{3}$ into (11.3). Then

$$
\begin{aligned}
\varphi=\beta_{3}^{\prime}-c^{2} \alpha_{3}\left(-2 \alpha_{1}\right)^{-1 / 2} & {\left[A_{3} v+\sum_{j=1}^{4} A_{3 j} \sin j v\right] } \\
& +\alpha_{3} \alpha_{2}^{-1} u^{1 / 2}\left\{( 1 - S ) ^ { - 1 / 2 } \left[\left(h_{1}+h_{2}\right)\left\{\frac{\pi}{2}(1+2 \zeta)^{-1 / 2}+\chi_{0}\right\}\right.\right. \\
+ & \left.\left.\left(h_{1}-h_{2}\right)\left\{-\frac{\pi}{2}(1+2 \zeta)^{-1 / 2}+\chi_{1}\right\}\right]+\left(\frac{\pi}{2}+\psi\right) B_{3}-\frac{3}{4} C_{1} C_{2} Q \cos \psi+\frac{3}{32} C_{2}^{2} Q^{2} \sin 2 \psi\right\} .
\end{aligned}
$$

Here $A_{3}$ is given by (5.61) through (5.65) in V1961b. Because of typographical errors there, I include here the correct expressions for $A_{32}$ and $A_{33}$ :

$$
\begin{aligned}
& A_{32}=\left(1-e^{2}\right)^{1 / 2} p^{-3}\left[\frac{e^{2}}{4}+\frac{3}{4} \frac{b_{1}}{p} e^{2}-p^{-2}\left(\frac{e^{4}}{4}+\frac{3}{2} e^{2}\right)\left(\frac{b_{2}^{2}}{2}+c^{2}\right)\right] \\
& A_{33}=\left(1-e^{2}\right)^{1 / 2} p^{-3}\left[\frac{e^{3} b_{1}}{12 p}-\frac{e^{3}}{3 p^{2}}\left(\frac{b_{2}^{2}}{2}+c^{2}\right)\right] .
\end{aligned}
$$

Here $b_{2}^{2}$ is simply the $B$ in (34.1) of this paper.

The symbols $h_{1}$ and $h_{2}$ are abbreviations:

$$
h_{1} \equiv \frac{1}{2}\left(1+C_{1}-C_{2}\right)^{-1 / 2} \quad(147.1) \quad h_{2} \equiv \frac{1}{2}\left(1-C_{1}-C_{2}\right)^{-1 / 2} .
$$

By (18), (21.2), (26), and (31), we find for $\alpha_{3}$

$$
\alpha_{3}=\left[\mu p_{0}(1-S)\right]^{1 / 2} \operatorname{sgn} \alpha_{3}\left[1-\frac{c^{2} S}{a_{0} p_{0}}-\frac{\left(\frac{2 \delta}{p_{0}}\right)^{2}\left(1-\frac{c^{2} S}{a_{0} p_{0}}\right) S}{\left[1+\frac{c^{2}}{a_{0} p_{0}}(1-2 S)\right]^{2}}\right]^{1 / 2} .
$$

The error here is of order $J_{2}^{4}$. With the same error, we can simplify this to

$$
\alpha_{3}=\left[\mu p_{0}(1-S)\right]^{1 / 2} \operatorname{sgn} \alpha_{3}\left[1-S\left\{\frac{c^{2}}{a_{0} p_{0}}+\left(\frac{2 \delta}{p_{0}}\right)^{2}\left(1-\frac{2 c^{2}}{a p}+\frac{3 c^{2} S}{a p}\right)\right\}\right]^{1 / 2}
$$


Alternatively, when $\alpha_{2}$ is known, we may write

$$
\alpha_{3}=\alpha_{2}\left(1-\frac{S}{u}\right)^{1 / 2} \operatorname{sgn} \alpha_{3} .
$$

In (146) the constant $\beta_{3}^{\prime}$ would reduce to $\Omega-\frac{1}{2} \pi \operatorname{sgn} \alpha_{3}$ in the Keplerian case, as one may see by solving the two body problem with lower limit $\eta_{1}\left(=-\eta_{0}\right)$ in the $\eta$-integrals. Also in (146), consider the constant term:

$$
\frac{1}{2} \pi \alpha_{3} \alpha_{2}^{-1} u^{1 / 2}(1-S)^{-1 / 2}(1+2 \zeta)^{-1 / 2}\left[h_{1}+h_{2}-\left(h_{1}-h_{2}\right)\right]+\frac{1}{2} \pi B_{3} .
$$

For $J_{2}=0$ and $J_{3}=0$, we have $u=1, \zeta=0, h_{1}+h_{2}=1, h_{1}-h_{2}=0, B_{3}=0, S=\sin ^{2} I$, and $\alpha_{3} \alpha_{2}^{-1}$ $=\cos I$. Thus it then becomes $\frac{1}{2} \pi \cos I|\sec I|=\frac{1}{2} \pi \operatorname{sgn} \alpha_{3}$. If we lump it into the constant $\beta_{3}^{\prime}$ and call the sum $\beta_{3}$, we then find that $\beta_{3}$ reduces, in the Keplerian case, to $\Omega$, the right ascension of the node.

Our amended equation thus takes the form

$$
\begin{aligned}
\varphi=\beta_{3}-c^{2} \alpha_{3}\left(-2 \alpha_{1}\right)^{-1 / 2}\left[A_{3} v+\sum_{j=1}^{4} A_{3 j} \sin j v\right]+\alpha_{3} \alpha_{2}^{-1} u^{1 / 2}\left\{( 1 - S ) ^ { - 1 / 2 } \left[\left(h_{1}+h_{2}\right) \chi_{0}\right.\right. \\
\\
\left.\left.\quad+\left(h_{1}-h_{2}\right) \chi_{1}\right]+B_{3} \psi-\frac{3}{4} C_{1} C_{2} Q \cos \psi+\frac{3}{32} C_{2}^{2} Q^{2} \sin 2 \psi\right\},
\end{aligned}
$$

where $\chi_{0}$ and $\chi_{1}$ are given by (114) and $E_{2}^{\prime}$ and $E_{3}^{\prime}$ in (114) by (104), with $e_{2}=Q(1-P)^{-1}$ and $e_{3}=Q(1+P)^{-1}$.

It is not difficult to verify that (150) reduces to (8.50) of V1961b in the limit $\delta=0$. A much more delicate check is to show that (150) makes $\varphi$ a constant in a polar orbit, with jumps of $\pi$ as the satellite goes over a pole.

As an orbit approaches being a polar orbit, $\alpha_{3} \rightarrow 0$ and $S \rightarrow 1$, so that

$$
\varphi \rightarrow \beta_{3}+\alpha_{3} \alpha_{2}^{-1} u^{1 / 2}\left[\left(h_{1}+h_{2}\right) \chi_{0}+\left(h_{1}-h_{2}\right) \chi_{1}\right](1-S)^{-1 / 2} .
$$

Now by (148), since $\left(\mu p_{0}\right)^{1 / 2}=\alpha_{2}$, we have for $S \rightarrow 1$

$$
\alpha_{3} \alpha^{-1} u^{1 / 2}(1-S)^{-1 / 2}=\operatorname{sgn} \alpha_{3}\left[1-\frac{c^{2}}{a_{0} p_{0}}-\frac{\left(2 \delta / p_{0}\right)^{2}}{1-\frac{c^{2}}{a_{0} p_{0}}}\right]^{1 / 2}
$$

since by (31) $u \rightarrow 1$ as $S \rightarrow 1$. Also, for $S=1$, we have by (32) that (152) becomes

$$
\alpha_{3} \alpha_{2}^{-1} u^{1 / 2}(1-S)^{-1 / 2}=\operatorname{sgn} \alpha_{3}\left[\left(1-C_{2}\right)^{2}-C_{1}^{2}\right]^{1 / 2}\left(1-C_{2}\right)^{-1 / 2} .
$$

To simplify the term in (151) involving the $\chi$ 's, note that by (102.2), (32.1), and (32.2)

$$
2 \zeta=\left(1-C_{2}\right)^{-1} C_{1} .
$$

Then, by (147) and (154)

$$
(1-2 \zeta)^{-1 / 2}=2 h_{2}\left(1-C_{2}\right)^{1 / 2} \quad(155.1), \quad(1+2 \zeta)^{1 / 2}=2 h_{1}\left(1-C_{2}\right)^{1 / 2}
$$


In (114) let us now abbreviate $E_{2}^{\prime}(\psi+\pi / 2)$ to $E_{2}^{\prime}$ and $E_{3}^{\prime}(\psi-\pi / 2)$ to $E_{3}^{\prime}$. Equations (114) and (155) then yield

$$
\begin{aligned}
& \chi_{0}=\left(1-C_{2}\right)^{1 / 2}\left(h_{2} E_{2}^{\prime}+h_{1} E_{3}^{\prime}\right) \\
& \chi_{1}=\left(1-C_{2}\right)^{1 / 2}\left(h_{2} E_{2}^{\prime}-h_{1} E_{3}^{\prime}\right),
\end{aligned}
$$

from which it follows that

$$
\begin{aligned}
\left(h_{1}+h_{2}\right) \chi_{0}+\left(h_{1}-h_{2}\right) \chi_{1}=2\left(1-C_{2}\right)^{1 / 2} h_{1} h_{2}\left(E_{2}^{\prime}+E_{3}^{\prime}\right) & \\
& =\frac{1}{2}\left(1-C_{2}\right)^{1 / 2}\left[\left(1-C_{2}\right)^{2}-C_{1}^{2}\right]^{-1 / 2}\left(E_{2}^{\prime}+E_{3}^{\prime}\right),
\end{aligned}
$$

after insertion into (157) of the expressions (147) for $h_{1}$ and $h_{2}$. On inserting (153) and (158) into (151), we then find that for a polar orbit

$$
\varphi \rightarrow \beta_{3}+\frac{1}{2}\left(E_{2}^{\prime}+E_{3}^{\prime}\right) \operatorname{sgn} \alpha_{3}
$$

To discuss this expression, note that $e_{2}=Q(1-P)^{-1}$ and $e_{3}=Q(1+P)^{-1}$ are both unity for a polar orbit, since by (32.3) $P=0$ when $S=1$ and since by (47) $Q$ then equals $S=1$.

To see how $E_{2}^{\prime}+E_{3}^{\prime}$ behaves as $S \rightarrow 1$, we have to discuss the function $E^{\prime}(f)$, where the argument $f$ plays the role of true anomaly and the function $E^{\prime}$ the role of eccentric anomaly.

Now by their definitions $E^{\prime}$ and $f$ agree in value at all multiples of $\pi$. Also

$$
\frac{d E^{\prime}}{d f}=\frac{\sqrt{1-e^{2}}}{1+e \cos f}
$$

Thus

$$
\begin{aligned}
\frac{d E^{\prime}}{d f} & =\left(\frac{1-e}{1+e}\right)^{1 / 2} \rightarrow 0 \text { as } e \rightarrow 1, \quad f=2 \tau \pi \\
& =\left(\frac{1+e}{1-e}\right)^{1 / 2} \rightarrow \infty \text { as } e \rightarrow 1, \quad f=(2 \tau+1) \pi
\end{aligned}
$$

The plot of $E^{\prime}$ versus $f$ for $e=1$ is thus as follows

FigURE 1. Plot of eccentric anomaly versus true anomaly for $\mathrm{e}=1$.

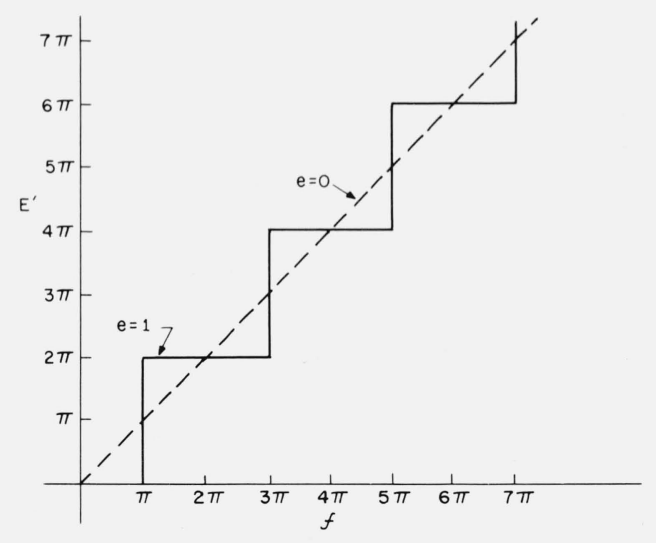


For $e=1$, one sees that $E^{\prime}(f)$ remains constant from $f=(2 \tau+1) \pi$ to $(2 \tau+3) \pi$, but then jumps by $2 \pi$.

Let us assume $\operatorname{sgn} \alpha_{3}=1$ and consider what happens as $\psi$ increases from $-\frac{\pi}{2}+\epsilon^{\prime}$ to $\frac{\pi}{2}-\epsilon^{\prime}$. In this range $\psi+\pi / 2$ increases from $\epsilon^{\prime}$ to $\pi-\epsilon^{\prime}$ and $\psi-\pi / 2$ from $-\pi+\epsilon^{\prime}$ to $-\epsilon^{\prime}$. In this range, therefore, both $E^{\prime}(\psi+\pi / 2)$ and $E^{\prime}(\psi-\pi / 2)$ remain constant, so that $\varphi$ remains constant, by (159). As $\psi$ increases beyond $\pi / 2$, however, $\psi+\pi / 2$ passes through an odd multiple of $\pi$ and $\psi-\pi / 2$ through an even multiple of $\pi$. Thus $E^{\prime}(\psi+\pi / 2)$ jumps by $2 \pi$ and $E^{\prime}(\psi-\pi / 2)$ remains constant, so that, by (159), $\varphi$ jumps by $\pi$. This is at the north pole. A similar jump takes place at the south pole. These results are of course trivial for a polar orbit, but they constitute an important test that the equation (150) for the right ascension had to pass.

\section{The Final Algorithm}

To summarize results, assume that $\mu, r_{e}, J_{2}, J_{3}$, and the orbital elements $a, e, S, l_{0}, g_{0}$, and $\beta_{3}$ are given. (Here $S$ is an element that would reduce to $\sin ^{2} I=\eta_{0}^{2}$ of V1961b, but with $J_{3}$ incorporated into the reference orbit it may go negative for orbits close to equatorial.)

To calculate the reference orbit, compute

$$
c^{2}=r_{e}^{2} J_{2}\left(1-\frac{1}{4} J_{3}^{2} J_{2}^{-3}\right), \delta=-\frac{1}{2} r_{e} J_{2}^{-1} J_{3}>0 \text { for } J_{3}<0 \quad p=a\left(1-e^{2}\right),
$$

$A$ by (40.1), and $B$ by (40.2). Then

$a_{0}=a-\frac{1}{2} A, p_{0}=a_{0}^{-1}\left(B+a p-2 A a-c^{2}\right), \alpha_{2}=\left(\mu p_{0}\right)^{1 / 2}, u$ by (31), $C_{2}$ by (32.2), $C_{1}$ by (31.1), $P$ by (32.3), $b_{1}=-\frac{1}{2} A, b_{2}=B^{1 / 2}, \alpha_{1}=-\frac{1}{2} \mu a_{0}^{-1}$. Then compute

$A_{1}, A_{2}, A_{3}, A_{11}, A_{12}, A_{21}, A_{22}, A_{23}, A_{24}, A_{31}, A_{32}, A_{33}, A_{34}$ by p. 198 of V1961b.

Now compute $Q=\left(P^{2}+S\right)^{1 / 2}, B_{2}$ by (65), $B_{1}^{\prime}$ by (76), $B_{3}$ by (95), $\zeta$ by (102.2),

$$
e_{2}=Q(1-P)^{-1} \quad e_{3}=Q(1+P)^{-1}
$$

$2 \pi \nu_{1}$ by (122.1), $2 \pi \nu_{2}$ by (122.2), and $l_{0}$ and $g_{0}$ by (123). Then

$$
\begin{gathered}
M_{s}=l_{0}+2 \pi \nu_{1} t, \quad \psi_{s}=l_{0}+g_{0}+2 \pi \nu_{2} t \\
e^{\prime}=\frac{a e}{a+b_{1}}<e<1 .
\end{gathered}
$$

Find $M_{s}+E_{0}$ by solving

$$
M_{s}+E_{0}-e^{\prime} \sin \left(M_{s}+E_{0}\right)=M_{s}
$$

Solve (132) for $v=M_{s}+v_{0}$ and (133) for $\psi_{0}$. Then find $M_{1}$ by (136.2) and $E_{1}$ by (137) and solve (132) for $v=M_{s}+v_{0}+v_{1}$. Find $\psi_{1}$ by (139) and then $M_{2}$ by (143.2) and $E_{2}$ by (143.1).

Solve (132) for $v=M_{s}+v_{0}+v_{1}+v_{2}$ and find $\psi_{2}$ by (145). Then

$$
E=M_{s}+E_{0}+E_{1}+E_{2}, v=M_{s}+v_{0}+v_{1}+v_{2}, \psi=\psi_{s}+\psi_{0}+\psi_{1}+\psi_{2}
$$


The spheroidal coordinates $\rho$ and $\eta$ are then given by

$$
\begin{aligned}
& \rho=a(1-e \cos E)=(1+e \cos v)^{-1} p \\
& \eta=P+Q \sin \psi .
\end{aligned}
$$

To find the right ascension $\varphi$, note first the correct formulas for $A_{32}$ and $A_{33}$ in (146.1) of this paper or on p. 198 of V1961b. Then compute $h_{1}$ and $h_{2}$ by (147) and $\alpha_{3}$ by (148). Define $E_{2}^{\prime}(y)$ by

$$
\cos E_{2}^{\prime}(y)=\frac{e_{2}+\cos y}{1+e_{2} \cos y} \quad \sin E_{2}^{\prime}(y)=\frac{\left(1-e_{2}^{2}\right)^{1 / 2} \sin y}{1+e_{2} \cos y}
$$

and the requirement that $E_{2}^{\prime}$ and $y$ shall be equal whenever $y$ is a multiple of $\pi$. Define $E_{3}^{\prime}(y)$ in the same way, with $e_{3}$ replacing $e_{2}$.

Then calculate $\chi_{0}$ and $\chi_{1}$ by (114) and $\varphi$ by (150). Having $\rho, \eta$, and $\varphi$, we may now find the rectangular and spheroidal coordinates by means of (1.1) and (1.2).

\section{Further Remarks}

The present inclusion of $J_{3}$ into the separable orbit has apparently led to new secular terms of order $J_{2}^{2}$. These terms, $-\frac{1}{2} C_{1} P+\frac{3}{16} C_{1}^{2} Q^{2}$ in $B_{2}$ and $-\frac{3}{8} C_{1}^{2}$ in $B_{3}$, are proportional to $J_{3}^{2} J_{2}^{-2}$ $=O\left(J_{2}^{2}\right)$ and lead to terms in $M_{s}, \psi_{s}$, and $\overline{\dot{\varphi}}$. Since no previous treatment of third harmonic perturbations has led to such secular terms, the result requires checking.

To do so, note that by (2) and (3) the present $c^{2}$ is given by

$$
c^{2}=c_{0}^{2}-\delta^{2}
$$

where $c_{0}^{2}=r_{e}^{2} J_{2}$. It turns out that the $\delta^{2}$ terms implicit in the new $c^{2}$ are just sufficient to cancel the new terms in $\delta^{2}$.

Since $c^{4}=c_{0}^{4}-2 c_{0}^{2} \delta^{2}+\delta^{4}$, terms of order $c^{4}$ cannot cancel terms in $\delta^{2}$. In expanding the fundamental frequencies it is thus sufficient to carry terms only through $c^{2}$ and $\delta^{2}$, in checking this point. A rather long calculation then shows that

$$
\begin{gathered}
\dot{M}_{s}=2 \pi \nu_{1}=n_{0}+O\left(c^{4}\right) \\
\dot{\psi}_{s}=2 \pi \nu_{2}=n_{0}\left[1+\frac{3}{4} \frac{c_{0}^{2}}{p^{2}}(4-5 S)\right]+O\left(c^{4}\right) \\
\dot{\varphi}=2 \pi \nu_{3}=n_{0} \operatorname{sgn} \alpha_{3}\left[1+\frac{3}{4} \frac{c_{0}^{2}}{p^{2}}(4-5 S)-\frac{3}{2} \frac{c_{0}^{2}}{p^{2}}(1-S)^{1 / 2}\right]+O\left(c^{4}\right) .
\end{gathered}
$$

Here $n_{0}=\mu^{1 / 2} a_{0}^{-3 / 2}$, the mean motion corresponding to $a_{0}=-\mu\left(2 \alpha_{1}\right)^{-1}$, where $\alpha_{1}$ is the total energy. This means that, if we solve the problem for given initial conditions without using the third harmonic and then re-solve it with inclusion of the third harmonic, there will arise no secular terms of order $J_{3}^{2} J_{2}^{-2}$, provided that we use the same initial total energy. In turn, this means that if we use the same initial coordinates, we must change the initial velocities slightly or that if we use the same initial velocities we must change the initial coordinates slightly.

It does not appear worth while to do the extra calculation that would give us final formulas manifestly free of secular terms of order $J_{3}^{2} J_{2}^{-2}$. If we use the present results, we shall obtain secular terms correct through order $J_{2}^{3}$; this limitation comes from the error of order $J_{2}^{4}$ in (31.2). To obtain this secular accuracy, we find from (122) and (150) that we should keep terms through order 
$J_{2}^{3}$ in $B_{2}$ and $B_{3}$ and through order $J_{2}^{2}$ in $B_{1}^{\prime}$. By improving the solution of the cubic (27), we could calculate secular terms to any order of accuracy. Periodic terms are correct through order $J_{2}^{2}$; they also may be carried to a higher order if the need ever arises.

To obtain the orbital elements one may proceed as follows. From initial values determine $\alpha_{1}, \alpha_{2}$, and $\alpha_{3}$ by the appropriate modifications of eqs (3.8), (3.9), and (3.10) of V1961. They are

$$
\begin{aligned}
& \alpha_{1}=\frac{1}{2} u_{i}^{2}-\mu\left(\rho_{i}+\eta_{i} \delta\right)\left(\rho_{i}^{2}+c^{2} \eta_{i}^{2}\right)^{-1} \\
& \alpha_{3}=r_{i}^{2} \cos ^{2} \theta_{i} \dot{\varphi}_{i}=x_{i} \dot{y}_{i}-y_{i} \dot{x}_{i} \\
& \alpha_{2}^{2}=\left(1-\eta_{i}^{2}\right)^{-1}\left[\left(\rho_{i}^{2}+c^{2} \eta_{i}^{2}\right)^{2} \dot{\eta}_{i}^{2}+\alpha_{3}^{2}-\left(1-\eta_{i}^{2}\right)\left(2 \alpha_{1} c^{2} \eta_{i}^{2}+2 \mu \eta_{i} \delta\right)\right] .
\end{aligned}
$$

Here the equation for $\alpha_{2}$ follows from (10.2), (13.2), (50), and (59.1) of V1959. The subscript $i$ means initial value and $u_{i}$ is the initial speed.

From the $\alpha$ 's one can determine $a_{0}, e_{0}, p_{0}$, and $i_{0}$ from eqs (21) and (28) of the present paper and then determine $a, e$, and $\eta_{0}=\sin I$ by means of the equations on pp. (172) and (173) of V1961b. Knowing $a, e$, and $I$ to this approximation, one can then determine the values of $\beta_{1}, \beta_{2}$, and $\beta_{3}$ or of $l_{0}, g_{0}$, and $\beta_{3}$ by fitting the values of $\rho, \eta$, and $\varphi$ at various times, still using the equations in V1961b.

To improve these elements, now calculate the orbit, with elements $a, e, S=\sin ^{2} I, l_{0}, g_{0}$, and $\beta_{3}$ by means of the present paper. Observe the residuals and use them, with the aid of least squares, to obtain a differential correction to improve the orbital elements. Here a word of caution is necessary. If the orbit is close to equatorial, $S$ may go negative. This is all right, since it is only $Q^{2}=P^{2}+S$ that cannot become negative, with the present method.

Then repeat the process, until the elements converge to fixed values. One or two iterations should suffice, according to Bonavito (1964).

The present method of accounting for $J_{3}$ ought to be an improvement over the perturbation theory of V1963, for two reasons. There appear no small denominators, such as those containing $e$ or $\sin I$ in the perturbation theory. All periodic terms are correct through order $J_{2}^{2}$.

\section{References}

Bonavito, N. L. (1964), NASA Report D2312.

Vinti, J. P. (1959), J. Res. NBS 63B (Math. and Math. Phys.), No. 2, 105-116.

Vinti, J. P. (196la), J. Res. NBS 65B (Math. and Math. Phys.), No. 2, 131-135.

Vinti, J. P. (1961b), J. Res. NBS 65B (Math. and Math. Phys.), No. 3, 169-201.

Vinti, J. P. (1961c), Astron. J. 66, 514-516.

Vinti, J. P. (1962), J. Res. NBS 66B (Math. and Math. Phys.), No. 1, 5-13.

Vinti, J. P. (1963), J. Res. NBS 67B (Math. and Math. Phys.), No. 4, 191-222.

Vinti, J. P. (1966), J. Res. NBS 70B (Math. and Math. Phys.), No. 1, 1-16.

(Paper 70B1-166) 